\title{
THE CALIBRATION AND DATA PRODUCTS OF GALEX
}

\author{
Patrick Morrissey, ${ }^{1}$ Tim Conrow, ${ }^{1}$ Tom A. Barlow, ${ }^{1}$ Todd Small, ${ }^{1}$ Mark Seibert, ${ }^{2}$ Ted K. Wyder, ${ }^{1}$ \\ Tamás Budavári, ${ }^{3}$ Stephane Arnouts, ${ }^{4}$ Peter G. Friedman, ${ }^{1}$ Karl Forster, ${ }^{1}$ D. Christopher Martin, ${ }^{1}$ \\ Susan G. Neff, ${ }^{5}$ David Schiminovich, ${ }^{6}$ Luciana Bianchi, ${ }^{7}$ José Donas, $^{4}$ Timothy M. Heckman, ${ }^{3}$ \\ Young-Wook Lee, ${ }^{8}$ Barry F. Madore, ${ }^{2}$ Bruno Milliard, ${ }^{4}$ R. Michael Rich, \\ Alex S. Szalay, ${ }^{3}$ Barry Y. Welsh, ${ }^{10}$ and Sukyoung K. YI \\ Received 2006 December 8; accepted 2007 May 16
}

\begin{abstract}
We describe the calibration status and data products pertaining to the GR2 and GR3 data releases of the Galaxy Evolution Explorer (GALEX). These releases have identical pipeline calibrations that are significantly improved over the GR1 data release. GALEX continues to survey the sky in the far-ultraviolet (FUV, $154 \mathrm{~nm}$ ) and near-ultraviolet (NUV, $\sim 232 \mathrm{~nm}$ ) bands, providing simultaneous imaging with a pair of photon-counting, microchannel plate, delay line readout detectors. These $1.25^{\circ}$ field of view detectors are well suited to ultraviolet observations because of their excellent red rejection and negligible background. A dithered mode of observing and photon list output pose complex requirements on the data processing pipeline, entangling detector calibrations, and aspect reconstruction algorithms. Recent improvements have achieved photometric repeatability of 0.05 and $0.03 m_{\mathrm{AB}}$ in the FUV and NUV, respectively. We have detected a long-term drift of order $1 \%$ FUV and $6 \%$ NUV over the mission. Astrometric precision is of order $0.5^{\prime \prime} \mathrm{rms}$ in both bands. In this paper we provide the GALEX user with a broad overview of the calibration issues likely to be confronted in the current release. Improvements are likely as the GALEX mission continues into an extended phase with a healthy instrument, no consumables, and increased opportunities for guest investigations.
\end{abstract}

Subject headings: space vehicles — surveys — telescopes — ultraviolet: general

Online material: color figures

\section{INTRODUCTION}

We describe the calibration status and data products pertaining to the combined GR2 and GR3 data releases of the Galaxy Evolution Explorer (GALEX). GALEX is a NASA Small Explorer mission performing an all-sky ultraviolet (UV) survey in the far$\mathrm{UV}(\mathrm{FUV} \sim 154 \mathrm{~nm})$ and near-UV (NUV $\sim 232 \mathrm{~nm}$ ) bands with $4-6$ " resolution and $\sim 50 \mathrm{~cm}^{2}$ effective area. At the time of this writing, over $13,500 \mathrm{deg}^{2}$ of this survey are available to the public via the Internet from the Multimission Archive at Space Telescope Science Institute (MAST). ${ }^{11}$

The GR2 and GR3 releases have identical pipeline calibrations that are significantly improved over the previous GR1 release. Photometric precision at a given epoch is 0.05 and $0.03 m_{\mathrm{AB}}$ in the FUV and NUV, respectively. We have detected a long-term

\footnotetext{
${ }^{1}$ California Institute of Technology, MC 405-47, 1200 East California Boulevard, Pasadena, CA 91125 .

2 Observatories of the Carnegie Institution of Washington, 813 Santa Barbara Street, Pasadena, CA 91101.

3 Department of Physics and Astronomy, The Johns Hopkins University, Homewood Campus, Baltimore, MD 21218.

${ }^{4}$ Laboratoire d'Astrophysique de Marseille, BP 8, Traverse du Siphon, 13376 Marseille Cedex 12, France.

5 Laboratory for Astronomy and Solar Physics, NASA Goddard Space Flight Center, Greenbelt, MD 20771.

6 Department of Astronomy, Columbia University, New York, NY 10027.

7 Center for Astrophysical Sciences, The Johns Hopkins University, 3400 North Charles Street, Baltimore, MD 21218.

${ }_{8}$ Center for Space Astrophysics, Yonsei University, Seoul 120-749, Korea.

9 Department of Physics and Astronomy, University of California, Los Angeles, CA 90095 .

${ }^{10}$ Space Sciences Laboratory, University of California at Berkeley, 601 Campbell Hall, Berkeley, CA 94720.

${ }_{11}$ See http://galex.stsci.edu.
}

drift of order $1 \%$ FUV and $6 \%$ NUV over the mission. The astrometric precision has been improved to $0.5^{\prime \prime} \mathrm{rms}$.

The path from raw telemetry to corrected catalogs, images, and spectra is a complicated one for the GALEX pipeline. This fact is illustrated by Figure 1, which shows a GALEX image in detector and sky coordinates, which are representative of the pre- and postpipeline images. This overview highlights the data quality, errors, and peculiarities that are likely to confront astronomers. As is true for any large data set, knowledge of these basic characteristics is essential to avoid confusion.

The GALEX mission continues into an extended phase with a healthy instrument, no consumables, and increased opportunities for guest investigations. While the survey is ongoing, it is likely that we will make improvements to the calibration and discover additional sources of error. We expect to provide updates to this paper on the Web, ${ }^{12}$ as well as in the literature.

This paper is organized as follows. In $\oint 2$ we summarize the design of the mission and instrument. In $\S 3$ we describe how GALEX data are reconstructed and what the key pipeline products are. In $\S 4$ we describe the photometric calibration of the data, including a discussion of saturation effects and drift. In $\S 5$ we describe the instrument resolution across the field of view and as a function of detector gain. In $\S 6$ we describe the astrometric performance of the instrument. In $\S 7$ we describe the spectroscopy calibration and present an example extraction. Finally, in $\S 8$ we summarize the key points of the paper.

\section{MISSION AND INSTRUMENT OVERVIEW}

GALEX was launched on an Orbital Sciences Corporation (Orbital) Pegasus rocket on 2003 April 28 at 12:00 UT from the

${ }^{12}$ See http://www.galex.caltech.edu. 

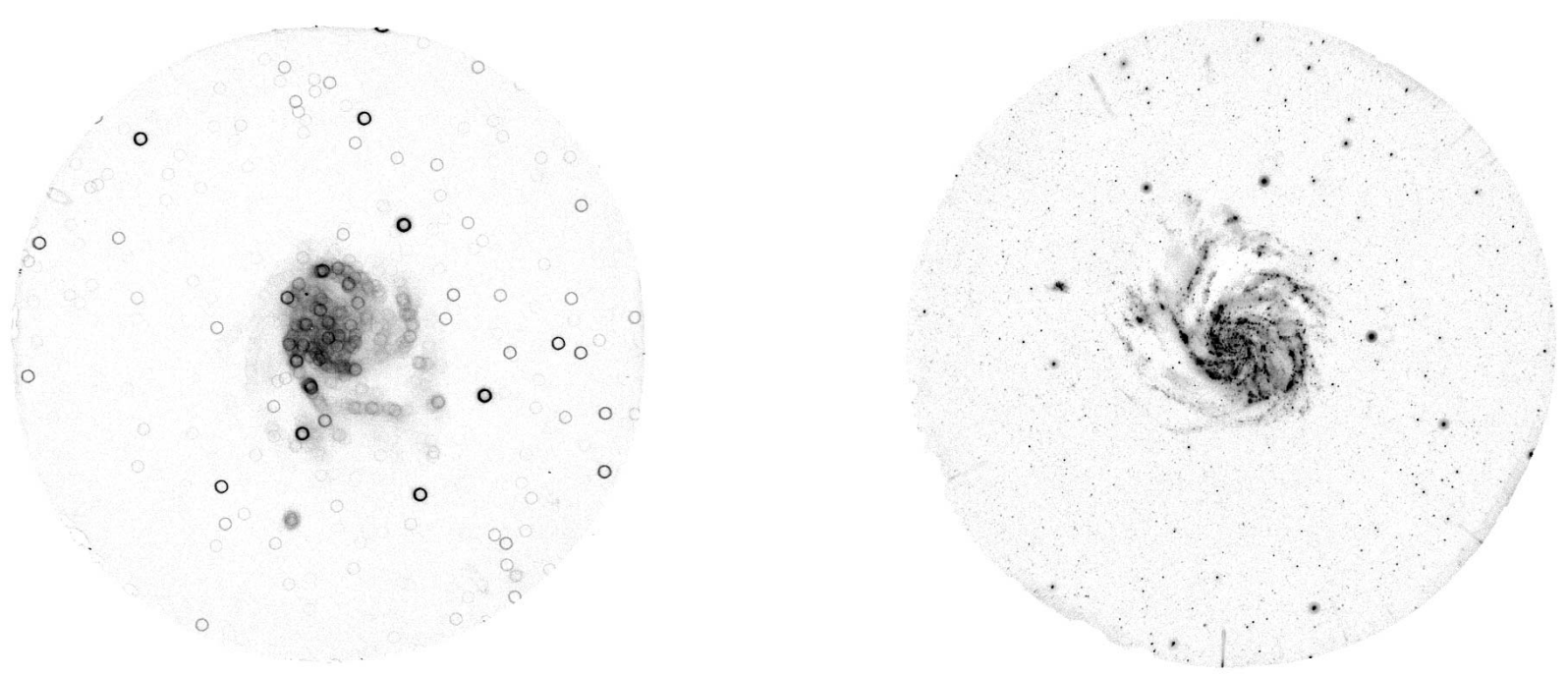

FIG. 1.-Left panel: A GALEXNUV "dose" image of M101 in spacecraft coordinates clearly showing the 1' dither pattern, which is used for all observations to smooth out the effects of the detector flat field and reduce MCP fatigue. Right panel: The fully processed, background-subtracted NUV image of M101 in sky coordinates, which provides the basis for the source catalog data.

Kennedy Space Center into a circular, $700 \mathrm{~km}, 29^{\circ}$ inclination orbit. This $0.5 \mathrm{~m}$ diameter instrument has a very wide $1.25^{\circ}$ field of view sensitive to $m_{\mathrm{AB}} \sim 21$ in the All Sky Imaging Survey (AIS), and to $m_{\mathrm{AB}} \sim 25$ in the Deep Imaging Survey (DIS). The instrument provides simultaneous FUV and NUV imaging with a pair of photon-counting, microchannel plate, delay line readout detectors. These detectors are well suited to ultraviolet observations because of their excellent red rejection and negligible background. An objective grism mode is also available for low-resolution spectroscopy. The last comparable survey in this wavelength range, which extended to $m_{\mathrm{AB}} \sim 9$, was performed by the Belgian/UK Ultraviolet Sky Survey Telescope on the TD1 satellite launched in 1972 and described by Boksenberg et al. (1973).

GALEX makes science observations on the night side of each orbit (to keep sky background to a minimum) during "eclipses" that are typically in the range $1500-1800 \mathrm{~s}$. In the AIS, as many as 12 targets are observed during an eclipse, while the Medium Imaging Survey (MIS) has one target per eclipse and the DIS returns to the same target for 30 or more eclipses. As of this writing, we have observed over 19,000 deg ${ }^{2}$ of sky and accumulated over 4 terabytes of science telemetry. An overview of key performance parameters is presented in Table 1.

A GALEX detector head is shown schematically in Figure 2. Individual photons incident at the cathode set off an isolated avalanche of current inside parallel $10-12 \mu \mathrm{m}$ diameter microchannel plate (MCP) pores as they accelerate through a few $\mathrm{keV}$ field. The resulting charge cloud at the rear of the MCP stack, approximately $10^{7}$ electrons in size, is deposited on a double-layer anode, divided, and finally measured at each of four outputs. The layers of the anode form a pair of orthogonal delay lines. Timing differences measured for the charge pulses at each corner of the anode are proportional to the position of the initiating photon event. For faint UV observations, the combination of low background (zero read noise) and high red rejection that these detectors possess represents a favorable trade-off against the superior QE and field flatness of more conventional CCD detectors. Furthermore, MCP detectors do not require cooling, an important consideration in the contamination-sensitive UV band. In order to improve photometric performance, observations are dithered in a spiral pattern approximately $1^{\prime}$ in diameter (at a rate of approximately 0.5 revolutions per minute) that smooth out small-scale variations in the flat field and reduce bright source fatigue on the MCPs.

A more detailed instrument overview is provided in Morrissey et al. (2005) while the mission design is described by Martin et al. (2005). Further details of our mission operations experience are described in Forster et al. (2006) and Morrissey (2006).

\section{IMAGE RECONSTRUCTION OVERVIEW}

The GALEX detector output is a list of time-ordered 40 bit photon positions and pulse heights. These data are delivered to the instrument Data Processing Unit (DPU), which adds a timing stamp every $5 \mathrm{~ms}$ (or every $20 \mu$ s in uncompressed mode). The time stamp is derived from the spacecraft clock, which has a drift of the order of $1 \mathrm{~s}$ each week that is corrected with ground updates. Data are stored in the $2.8 \mathrm{~GB}$ ring buffer of a Solid State Recorder (SSR), which is downlinked to a ground station approximately four times each day.

The ground pipeline reconstructs a list of photon positions and arrival times into an image. Broadly speaking, the photon lists are corrected for detector effects and image motion, projected into sky coordinates, binned into images, flat-fielded, and finally background-subtracted. These final image products are then scanned by the open-source SExtractor program (Bertin \& Arnouts 1996) to produce catalogs of source positions and fluxes. The steps along this path are described in more detail in the following sections.

\subsection{Static Calibration}

The static calibrations are those used to assemble raw detector event positions into accurate locations in the GALEX focal plane. They also flag events that are not photon generated, such as the periodic "STIM" pulses that are always electronically superimposed (in parallel with real photon events) on the detector anodes, and regions on the MCPs that are dominated by hotspots.

Each photon event in the GALEX science telemetry is a heavily encoded group of fields that, when decoded and assembled, produces key values listed in Table 2. In the GALEX design (Jelinsky et al. 2003), a free-running coarse clock subdivides the large detector areas into an $8 \times 8$ grid. Detector event pulses are measured asynchronously while this clock runs, so that each position (proportional to the measured arrival time difference of 
TABLE 1

Summary of Measured Performance Parameters for GaleX

\begin{tabular}{|c|c|c|}
\hline Item & FUV Band & NUV Band \\
\hline Bandwidth $^{\mathrm{a}} \ldots \ldots \ldots \ldots \ldots \ldots \ldots \ldots$ & $1344-1786 \AA$ & $1771-2831 \AA$ \\
\hline 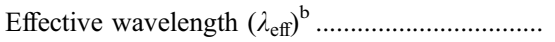 & $1538.6 \AA$ & $2315.7 \AA$ \\
\hline 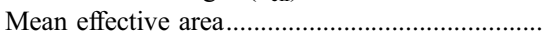 & $19.6 \mathrm{~cm}^{2}$ & $33.6 \mathrm{~cm}^{2}$ \\
\hline 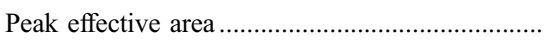 & $36.8 \mathrm{~cm}^{2}$ at $1480 \AA$ & $61.7 \mathrm{~cm}^{2}$ at $2200 \AA$ \\
\hline 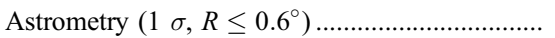 & $\pm 0.59^{\prime \prime}$ & $\pm 0.49^{\prime \prime}$ \\
\hline 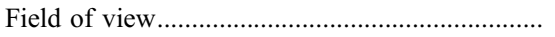 & $1.27^{\circ}$ & $1.25^{\circ}$ \\
\hline 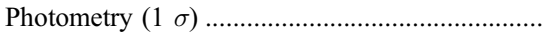 & $\pm 0.05 m_{\mathrm{AB}}$ & $\pm 0.03 m_{\mathrm{AB}}$ \\
\hline 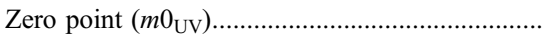 & 18.82 & 20.08 \\
\hline 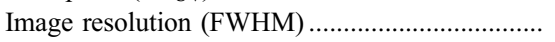 & $4.2^{\prime \prime}$ & $5.3^{\prime \prime}$ \\
\hline Spectral resolution $(\lambda / \Delta \lambda) \ldots \ldots \ldots \ldots \ldots$ & 200 & 118 \\
\hline 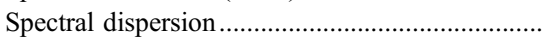 & $1.64 \AA \operatorname{arcsec}^{-1}$ & $4.04 \AA \operatorname{arcsec}^{-1}$ \\
\hline \multicolumn{3}{|l|}{ Detector background (typical): } \\
\hline Total ........................................ & 78 counts $\mathrm{s}^{-1}$ & 193 counts $\mathrm{s}^{-1}$ \\
\hline 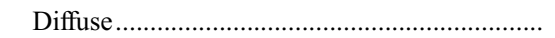 & 0.66 counts $\mathrm{s}^{-1} \mathrm{~cm}^{-2}$ & 1.82 counts $\mathrm{s}^{-1} \mathrm{~cm}^{-2}$ \\
\hline 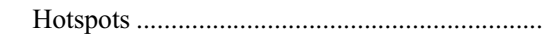 & 47 counts $s^{-1}$ & 107 counts $\mathrm{s}^{-1}$ \\
\hline Sky background (typical) ${ }^{\mathrm{c}} \ldots .$. & 1000 counts $\mathrm{s}^{-1}$ & 10,000 counts $s^{1}$ \\
\hline \multicolumn{3}{|l|}{ Limiting magnitude $(5 \sigma)$ : } \\
\hline 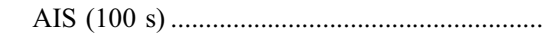 & 19.9 & 20.8 \\
\hline 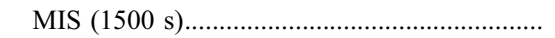 & 22.6 & 22.7 \\
\hline 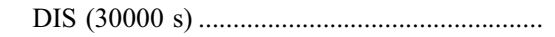 & 24.8 & 24.4 \\
\hline \multicolumn{3}{|l|}{ Linearity: } \\
\hline 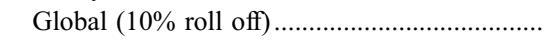 & \multicolumn{2}{|c|}{18,000 counts $\mathrm{s}^{-1}$} \\
\hline 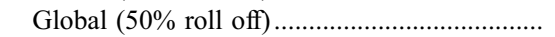 & \multicolumn{2}{|c|}{91,000 counts $\mathrm{s}^{-1}$} \\
\hline 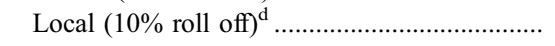 & $114{\text { counts } \mathrm{s}^{-1}}^{-1}$ & 303 counts $s^{-1}$ \\
\hline 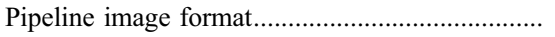 & \multicolumn{2}{|c|}{$3840 \times 3840$ elements with $1.5^{\prime \prime}$ pixels } \\
\hline
\end{tabular}

${ }^{a}$ Includes wavelengths with effective area at least $10 \%$ of the peak value.

b Wavelength-weighted: $\lambda_{\text {eff }}=\int A_{\text {eff }} \lambda / \int A_{\text {eff }}$.

c These correspond to 246 and 601 photons s${ }^{-1} \mathrm{~cm}^{-2} \mathrm{sr}^{-1} \AA^{-1}$, respectively.

${ }^{\mathrm{d}}$ These are worst-case values for point sources.

pulses from either side of the delay line anode on each axis) comprises an integer number of coarse clock steps plus a fine position (essentially the coarse clock phase).

In order to construct a raw source position $(X, Y)$ from these data, one would apply the following formulae:

$$
\begin{gathered}
X=X_{\mathrm{AmC}}+\alpha X_{B}, \\
Y=Y_{\mathrm{AmC}}+\beta Y_{B},
\end{gathered}
$$

where the constants $\alpha$ and $\beta$ are in the range of 2000 (solved by minimizing source widths across the field of view), $X_{B}$ and $Y_{B}$ are the coarse clock counters, and $X_{\mathrm{AmC}}$ and $Y_{\mathrm{AmC}}$ are the "fine" position measurements representing the coarse clock phase. ${ }^{13}$ After the detector positions are assembled, the following static

\footnotetext{
13 The notation derives from the ends of the delay line "A" and " $\mathrm{C}$," or sometimes "START" and "STOP" in other work, thus the position is proportional to a timing difference of $\mathrm{A}-\mathrm{C}$ or "AmC."
}

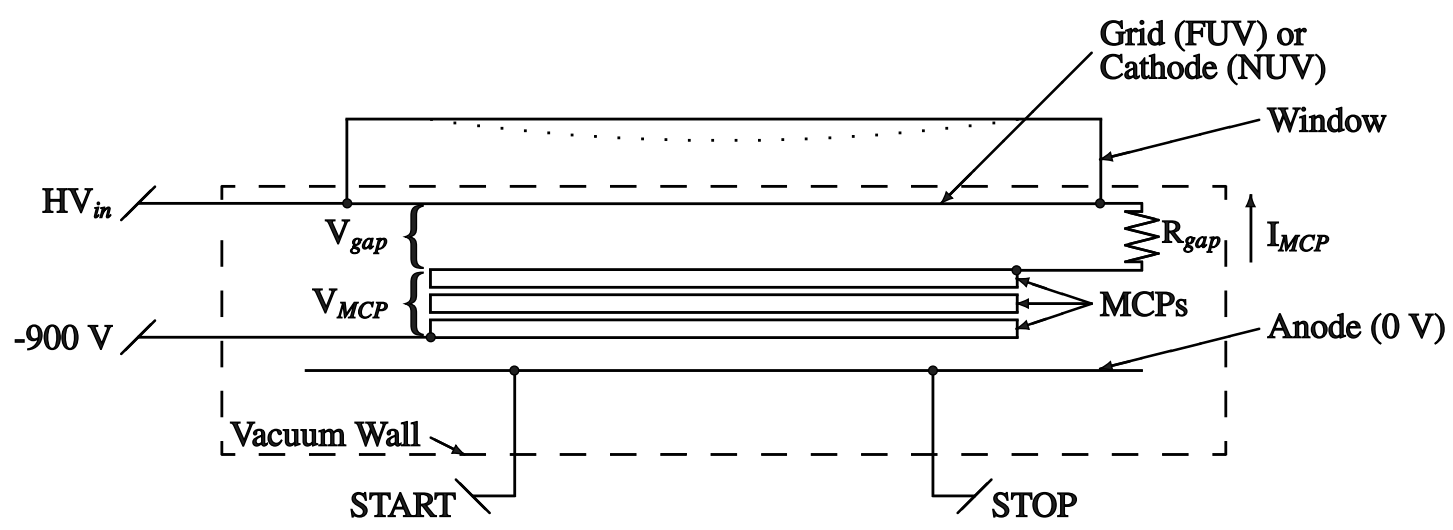

FIG. 2.-GALEX sealed tube detector head electromechanical block diagram. The NUV and FUV tube heads are nearly identical with the principal differences being the choice of photocathode material (CsI for FUV and $\mathrm{Cs}_{2} \mathrm{Te}$ for NUV) and window material $\left(\mathrm{MgF}_{2}\right.$ for FUV, $\mathrm{SiO}_{2}$ for NUV). Also, the cathode material is deposited on the detector window in the NUV channel but directly on the MCP in the FUV channel. Instead of a cathode, the FUV window has a charged grid of wires that enhances the sensitivity of the detector. The NUV cathode is proximity focused on the front MCP, and due to its reentrant design is more prone to reflections from off-field sources. $H V_{\text {in }}$ represents the negative applied voltage, 5-6 keV, while a resistor chain $\left(R_{\text {gap }} \sim 10 \mathrm{MOhm}\right.$ in series with the MCPs at about $30 \mathrm{MOhm}$ each $)$ sets the voltage across the gap $\left(V_{\text {gap }} \sim 500 \mathrm{~V}\right)$ and MCP stack $\left(V_{\mathrm{MCP}} \sim 4500 \mathrm{~V}\right)$ driving a detector bias current $I_{\mathrm{MCP}} \sim 50 \mu \mathrm{A}$. The detector pulse outputs in one dimension are labeled "START" and "STOP" (or sometimes "A" and "C"). 
TABLE 2

Key Data Contained in the GaLeX RAW6 Format Photon Lists

\begin{tabular}{rrl}
\hline Column & Bits & \multicolumn{1}{c}{ Description } \\
\hline & \multicolumn{1}{c}{ Event detection time } \\
\hline
\end{tabular}

Note.-Photons from the detector itself are not time stamped but rather time ordered, and the time stamp is added by the DPU before being written to the SSR.

${ }^{\mathrm{a}}$ In high-resolution mode, the timing precision uses 40 bits to attain $20 \mu \mathrm{s}$ resolution; however, this mode is rarely used. For most data, only 32 bits are significant, attaining $5 \mathrm{~ms}$ resolution.

corrections are applied (in order) to correct the raw positions to a linear representation of the actual source position in the focal plane:

Centering and scaling.-Coarsely scales the raw positions from bins to microns. The scale factors are different for each axis.

Wiggle.-The GALEX coarse-bit subarray approach allows degenerate combinations of coarse and fine components for a given detector location. Each of these may have a slightly different electronic nonlinearity correction owing to the differing phases of the time-to-amplitude converter (TAC) for each event. The image of a single point source on the detector is thus blurred by the superposition of all of the TAC data, an effect referred to as wiggle. This contrasts with smaller, more conventional time-to-amplitude conversion systems. These do not utilize a coarse counter bit and translate all electronic nonlinearities into source position errors rather than source widths. With careful part selection, "wiggle" in the flight units has been minimized considerably. In order to eliminate it completely, each detected event includes a 5 bit value $X_{\mathrm{A}}$, which represents where in the TAC range each measurement was made. Residual electronic nonlinearities are then removed using lookup tables for combinations of $\left(X, X_{\mathrm{A}}\right)$ and $\left(Y, X_{\mathrm{A}}\right)$. Both axes use the same $X_{\mathrm{A}}$ data because the $X$ and $Y$ pulses are correlated in time for a given detector position.

Walk.-This transformation corrects position versus pulse height $(Q)$ errors. A lookup table correction applied in a similar manner as wiggle, reflective of the fact that the electronics cannot perfectly compensate for pulse sizes that vary by a factor of 10 across the detector.

Spatial nonlinearity (distortion).-A lookup table corrects high-voltage detector edge effects, which cause electrons to bend from their optimal path, distorting the image especially near the edge of the field.

Hotspot masking. - Each detector has numerous small, high count rate features resulting from a combination of microscopic MCP debris and channel defects that combined generate about half of the intrinsic detector background. These spots come and go and are best handled by direct masking, which eliminates only about a percent of the detector area. New masks are generated roughly annually; hotspots are generally easy to pick out of images because they "reverse dither" (since they are fixed in detector space) and create $\sim 1^{\prime}$ circular artifacts in GALEX sky-space images. In the data pipeline, the hot spot masks create localized regions of zero effective sensitivity in detector space, one reason why dithered observations are preferred for these detectors. Depending on pointing accuracy in a given observation, the dither typically maps these regions into "donuts" of reduced (but not zero) response on the sky.

\subsection{Dynamic Aspect Correction}

The fully corrected event positions in spacecraft coordinates accurately record where each photon landed in the GALEX focal plane. The next step is to reconstruct the spacecraft pointing on the sky as a function of time in order to remove the dither pattern from the observed data. This is the three-parameter "aspect," which includes the right ascension, declination, and roll of the telescope boresight. For imaging mode data, a precise estimate is arrived at by refining the spacecraft Attitude Control System (ACS) pointing estimate with NUV data ${ }^{14}$ binned into $1 \mathrm{~s}$ slices. These snapshot images are each matched to the ACT catalog (Urban et al. 1998) of known stars, ${ }^{15}$ and the average error vector from the matches to the catalog stars are used to establish a correction to apply to the ACS estimate. The source positions are then transformed to sky coordinates (J2000.0) using a Gnomonic projection based on the pointing at the interpolated time.

Grism data undergoes a similar process, but the software cannot match spectra to known stars at this stage of processing. A relative aspect solution is performed based solely on the instantaneous spectral image positions, and astrometric refinement is added at a later stage.

Once sky coordinates have been assigned to each photon, an "extended photon list" is computed for internal use by the pipeline in forming the final data products. In cases where detailed, time-dependent behavior of one or more objects over the course of an exposure is of interest, the extended photon list can be written out to a so called "x-file" by special request.

\subsection{Pipeline Products}

Images and catalogs from various stages of pipeline processing reveal many characteristics of the complex, multidimensional $G A L E X$ data set and are included with each data release as pipeline products. A description of key products is provided here for reference.

The corrected photon data are binned into images and flux calibrated in order to form the basis for the GALEX catalogs. Count images (-cnt), which contain aspect-corrected detector counts per sky pixel are scaled by the effective area and exposure time to form the flux-calibrated intensity (-int ) images. This step is accomplished with the relative response (-rr) image, which contains the effective exposure for each pixel:

$$
r r(X, Y)=\Sigma_{t=0}^{T} \text { flat }[X(t), Y(t)] \times\left[1-f_{\text {dead }}(t)\right] \times d t(t)
$$

where flat $[X(t), Y(t)]$ is the detector flat field ${ }^{16}$ shifted to properly align with the dithered image time slice, $f_{\text {dead }}$ is the global electronic dead time based on the measured STIM pulser rate during each time slice, ${ }^{17}$ and $d t(t)$ is the fraction of each $1 \mathrm{~s}$ time

\footnotetext{
14 The process will run with FUV-only data, although owing to the generally decreased numbers and brightnesses of stars in FUV data, some parameters such as the interpolation interval need to be adjusted.

15 Occasionally, matches to known stars cannot be found, usually due to pointing errors. In these rare cases, a good point spread function (PSF) is achieved by performing the aspect refinement relative to any sources of emission detected in the field that are reasonably compact. This is called relative aspect refinement mode and results in images with good PSFs but poor absolute astrometry. Roll is not refined in relative mode.

16 The flat field has a value of 1 in regions for which a zero-point-magnitude source generates exactly 1 deadtime-corrected photon $\mathrm{s}^{-1}$.

17 Since the electronic STIM pulses occur at a precisely known rate, the fraction that are missing from the event list over a given interval will be known. This is the fractional dead time, which corrects the global rates only and does not account for local MCP saturation.
} 
TABLE 3

A Summary of Gale $X$ Pipeline Product Array Content and Format

\begin{tabular}{|c|c|c|c|c|c|}
\hline Product & Extension & Format & Units & Scale & Description \\
\hline Background-subtracted intensity.......... & -intbgsub & $3840 \times 3840$ & counts $\mathrm{s}^{-1}$ & $1.5^{\prime \prime} \mathrm{pixel}^{-1}$ & Intensity-sky background \\
\hline Dose & -scdose & $2250 \times 2250$ & counts & $3^{\prime \prime} \mathrm{pixel}^{-1}$ & Detector space, corrected for walk, wiggle, and distortion \\
\hline 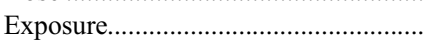 & $-\exp$ & $960 \times 960$ & $\mathrm{~s}$ & $6^{\prime \prime}$ pixel $^{-1}$ & Exposure, uncorrected for flat field or dead time \\
\hline 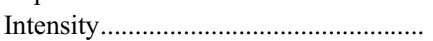 & -int & $3840 \times 3840$ & counts $\mathrm{s}^{-1}$ & $1.5^{\prime \prime} \mathrm{pixel}^{-1}$ & Count image divided by relative response image \\
\hline 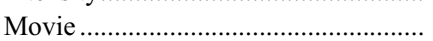 & -movie & $480 \times 480$ & counts & $12^{\prime \prime} \mathrm{pixel}^{-1}$ & Count map slices at $16 \mathrm{~s}$ intervals \\
\hline 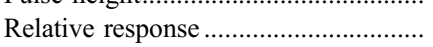 & $-\mathrm{rrhr}$ & $3840 \times 3840$ & $\mathrm{~s}$ & $1.5^{\prime \prime} \mathrm{pixel}^{-1}$ & Effective exposure, corrected for dither, flat field, and dead time \\
\hline 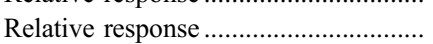 & $-\mathrm{rr}$ & $960 \times 960$ & $\mathrm{~s}$ & $6^{\prime \prime} \mathrm{pixel}^{-1}$ & Effective exposure, corrected for dither, flat field, and dead time \\
\hline 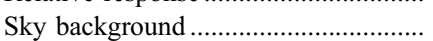 & -skybg & $3840 \times 3840$ & counts $\mathrm{s}^{-1}$ & $1.5^{\prime \prime} \mathrm{pixel}^{-1}$ & Sky background estimate \\
\hline 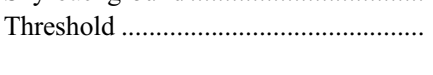 & $-w t$ & $3840 \times 3840$ & & $1.5^{\prime \prime}$ pixel $^{-1}$ & $\begin{array}{l}\text { Background-subtracted intensity map divided by } \\
\text { estimated Poisson noise }\end{array}$ \\
\hline
\end{tabular}

slice during which data were collected (i.e., HV was on and the detector was otherwise in a nominal observing mode). Building the effective exposure time in this way properly handles the fact that the edges of the image receive less exposure than the middle and that the sources each sample a $1^{\prime}$ region on the flat field. The high-resolution relative response image (-rrhr), which properly matches the count and intensity map pixel scales, is up-sampled from the 6 " relative response array. The intensity image is simply the count image divided by the high-resolution relative response image.

As a final step, the background in each intensity image must be estimated and removed. Since the GALEX detectors have negligible intrinsic background and the UV sky is dark, estimation of the true background is not properly handled by the code within SExtractor. Instead, we create a custom threshold image for SExtractor that allows a bypass of the normal process for distinguishing low rate sources from background. The typical sky background count rates in high Galactic latitude GALEX pointings are $\sim 10^{-4}\left(\sim 10^{-3}\right)$ photons $\mathrm{s}^{-1} \operatorname{arcsec}^{-2}$ in the FUV (NUV), thus it is necessary to use the full Poisson distribution. The background is determined in a set of large bins, $192^{\prime \prime}$ on a side. In images with exposure times greater than $10,000 \mathrm{~s}$, the bins are $96^{\prime \prime}$ on a side. For the Poisson distribution, the probability of observing greater than or equal to $k$ events for a mean rate $x$ is given by the incomplete gamma function $P_{k}(x)$ (Press et al. 1992, p. 216). The average is computed iteratively within each background bin by clipping out high pixels where $P_{k}(x)<1.35 \times$ $10^{-3}$, a probability equivalent to $3 \sigma$ for a Gaussian distribution. Even after clipping, bright or extended sources can still bias the background determination, therefore, a $5 \times 5$ median filter is applied to the background bins. The resulting background values are linearly interpolated to the full resolution of the images. We have determined that the background may be biased by a few percent due to contamination from the wings of objects. To address this problem, background estimation and source extraction software is run twice on each GALEX image. The second run uses source position data from the first iteration to mask out source pixels and recompute the sky background and background-subtracted intensity images. The resulting background image (-skybg) is subtracted from the intensity image to form the backgroundsubtracted intensity image (-intbgsub), which is the image scanned by SExtractor.

There remain issues with the background at the 5\% level that affect the MIS survey at the faint end. Furthermore, there are numerous artifacts not removed by the current pipeline that the user should be alert for. These include bright star glints in the NUV images near the edge of the detector window; large out-of-focus pupil images resulting from reflections in the imaging window and dichroic beam splitter; a 1' diameter skirt around bright NUV sources (at the few percent level in total energy) resulting from the proximity-focused photocathode; and photoemission and diffraction from the quantum-efficiency (QE) enhancing grid wires on the FUV detector window that appear as linear features around bright sources. Work is being done to flag and remove these features automatically in the pipeline software, but at present they amount to a small fraction of the survey coverage area $(\sim 1 \%)$.

The primary pipeline image products and descriptions are listed in Table 3, while catalog products are listed in Table 4. All of the catalog output products are generated by SExtractor, with the exception of the very useful merged catalog (-mcat), which combines FUV and NUV data in one table and adds derived columns including the detector coordinates of a source, detector intrinsic pulse height, number and brightness of nearby neighbors to a source, and many more. Matches between the bands are made by assigning a match probability to each pair of sources using the source separations and positional uncertainties. Sources with signal-to-noise $(\mathrm{S} / \mathrm{N})$ ratio of less than 2 or a separation greater

TABLE 4

Binary Tables and Related Products Generated by the GaLEX Pipeline

\begin{tabular}{|c|c|}
\hline Extension & Description \\
\hline -cat............................... & Primary SExtractor source catalogs for each band \\
\hline -fcat ............................... & SExtractor catalog of NUV extractions based on FUV coordinates \\
\hline -gauss .............................. & Listing of the Gaussian filter elements used by SExtractor for image convolution \\
\hline 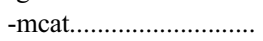 & Merged catalog for both NUV and FUV extractions \\
\hline 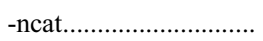 & SExtractor catalog of FUV extractions based on NUV coordinates \\
\hline -sexcols.......................... & Listing of SExtractor catalog column names \\
\hline -sexparams ................... & Listing of SExtractor parameters used \\
\hline
\end{tabular}


than $3^{\prime \prime}$ are not matched across bands (but do appear in the merged catalog). GALEX fields are uncrowded enough that the probability of a mismatch is very small; however, a multiple-candidate chain technique is used to assure that candidates are always matched the same way regardless of the order in which the process is started.

The GALEX data products are made available to the general public through the GALEX Science Database at MAST. A dedicated Web site provides access to the pipeline output files via a Web-based browser, as well as other derived products, such as JPEG images. The catalog data are also stored in a commercial SQL Server database engine fine-tuned for astronomy applications, which provides sophisticated query interfaces supporting, for example, efficient spatial and color searches. The GALEX MAST database also contains enhancements over the photometric pipeline outputs, e.g., spatial cross-matching to the Sloan Digital Sky Survey (SDSS) catalog. Also, since adjacent GALEX fields overlap slightly, a primary resolution algorithm deals with the problem of unique sample selection. The formal GALEX footprint definitions of the GALEX surveys are published in the National Virtual Observatory's online Footprint Service (Budavari et al. 2007). On this site, ${ }^{18}$ one can not only search and visualize the $G A L E X$ sky coverage, but also compute the exact area in square degrees or intersect it with other surveys.

\section{PHOTOMETRY}

\subsection{GALEX Magnitude Reference System}

The GALEX photometric calibration is tied to the Hubble Space Telescope (HST) through reference spectra supplied in the CALSPEC database and described by Bohlin et al. (2001). Almost all of the white dwarfs in this database are too bright for a photon-counting instrument such as GALEX in direct imaging mode. As such, we are restricted to the dimmest sources in the catalog, which are all ultimately based on the International Ultraviolet Observer (IUE) calibration. All of these data have been reprocessed for the $H S T$ calibration program, as detailed in Bohlin (1996). The GALEX calibration is thus traceable to a hydrogen white dwarf model scaled using the ground-based photometry of Landolt (1992) in combination with several white dwarf transfer standards measured with the HST Faint Object Spectrograph. The combination of models and measurements was used to define corrections to the $I U E$ calibration, thus transferring the $H S T$ calibration and allowing a fairly large group of standards from the IUE library to enter the CALSPEC database. Of these, we have observed 18 as detailed in Table 5; however, we use one of the dimmest, LDS749b, as our primary standard for direct imaging. For the most part this white dwarf is in the linear range of the GALEX detectors - bright enough for high $\mathrm{S} / \mathrm{N}$ measurements in just a few minutes, but dim enough not to saturate the microchannel plates. At the edge of the field in the NUV, where the detector gain is lowest, we apply a $\sim 20 \%$ correction for local saturation. This effect was discovered relatively recently as our database of white dwarf observations has expanded to include a much finer sampling of the detectors in regions where the gain is an issue. ${ }^{19}$

\footnotetext{
${ }_{18}$ See http://voservices.net/footprint.

19 Since the detector MCPs are clamped at the edges, there is less chance for a charge packet at the edge of the field to spread laterally while traversing the stack. At the detector center, where there are small gaps between the plates, charge can spread across the pores. Thus, the edges of the detectors tend to saturate more quickly than the centers. This effect can be seen for bright objects with multiple measurements by plotting the observed magnitude against the unsaturated detector pulse height, \#uv_q, tabulated in the merged catalog for each source. This quantity is the normal unsaturated pulse height of the detector at the location the source was measured. Note that the effect is minimized for large measuring apertures because the core of the PSF is primarily affected.
}

TABLE 5

HST White Dwarf Standards Observed by GaLEX

\begin{tabular}{|c|c|c|c|c|}
\hline Star & $m_{\mathrm{FUV}}{ }^{\mathrm{a}}$ & $m_{\mathrm{NUV}}{ }^{\mathrm{a}}$ & $\alpha(\mathrm{J} 2000.0)^{\mathrm{b}}$ & $\delta(\mathrm{J} 2000.0)^{\mathrm{b}}$ \\
\hline GD50 $\ldots$ & 11.98 & 12.57 & 34850.4 & -1126.8 \\
\hline HZ4 .. & 14.53 & 14.50 & 35522.0 & 94718.2 \\
\hline HZ2. & 12.86 & 13.25 & 41243.6 & 115149.9 \\
\hline G191B2B .. & & 10.17 & 5530.7 & 524949.3 \\
\hline GD108 & 12.39 & 12.77 & 10047.3 & -82628.5 \\
\hline HZ21 …………........ & 12.55 & 13.13 & 121356.3 & 325630.8 \\
\hline GD153 ……............. & 11.33 & 11.91 & 12572.4 & 22153.6 \\
\hline HZ43 .. & 10.75 & 11.36 & 131621.8 & 29557.8 \\
\hline HZ44 .. & 10.02 & 10.27 & 132335.0 & 36758.8 \\
\hline GRWp70d5824 .............. & 12.14 & 12.75 & 133849.2 & 70174.6 \\
\hline SAP041C $\ldots \ldots \ldots \ldots \ldots \ldots \ldots$ & & 16.69 & 145158 & $7143 \quad 17.4$ \\
\hline $\mathrm{BD}+33^{\circ} 2642 \ldots \ldots \ldots \ldots$ & 10.51 & 10.47 & 155159.9 & 325654.8 \\
\hline SAP177D .......................... & $\ldots$ & 18.35 & 155913.5 & 473641.4 \\
\hline SAP330E & $\ldots$ & 17.84 & 163133.8 & 30846.3 \\
\hline $\operatorname{LDS749B}^{\mathrm{c}} \ldots \ldots \ldots \ldots \ldots$ & 15.57 & 14.71 & 213216.4 & 01515.0 \\
\hline G93-48 & 12.14 & 12.39 & 215225.2 & 22317.9 \\
\hline NGC7293 ................... & 10.93 & 11.70 & 222938.5 & -21947.9 \\
\hline LTT9491 ........................ & 16.09 & 14.58 & 231935.4 & -185432.0 \\
\hline
\end{tabular}

${ }^{a}$ Magnitudes shown are predictions based on the GALEX bandpass and publicly available reference spectra from the HST CALSPEC database at http://www .stsci.edu/instruments/observatory/cds/calspec.html.

${ }^{\mathrm{b}}$ Coordinates are $G A L E X$-measured in the NUV. One exception is $\mathrm{BD}+33^{\circ} 2642$, which was only observed in grism mode; the coordinate provided is from Bohlin et al. (2001). Units of right ascension are hours, minutes, and seconds, and units of declination are degrees, arcminutes, and arcseconds.

c This white dwarf is the primary GALEX standard.

At the time of this writing we are planning observations of the (dimmest) CALSPEC white dwarf, LB227, in order to improve our confidence in the NUV zero point. Unfortunately, this source is not much dimmer than LDS749b, but since we believe the saturation of LDS749b is mild it should provide very useful verification of our saturation correction.

\subsection{Magnitude Zero Point}

GALEX uses the AB magnitude system of Oke \& Gunn (1983) with the FUV and NUV magnitudes defined as follows:

$$
m_{\mathrm{UV}}=m 0_{\mathrm{UV}}-2.5 \log f_{\mathrm{UV}}
$$

Here $f_{\mathrm{UV}}$ is the dead-time-corrected count rate for a given source divided by the flat-field map, which has a median value of order 0.85 , and $m 0_{\mathrm{UV}}$ is the zero point that corresponds to the $\mathrm{AB}$ magnitude of a 1 count $\mathrm{s}^{-1}$ flat-field-corrected detection. There are independent relative response functions, and the zero points are

$$
\begin{aligned}
& m 0_{\mathrm{FUV}}=18.82, \\
& m 0_{\mathrm{NUV}}=20.08 .
\end{aligned}
$$

The GALEX project has maintained these zero points constant; they are based on the imaging-mode bandpass determined during ground calibration. Improvements in calibration have been incorporated by adjusting the flat field, which is a stacked sky background image modified by an interpolated grid of white dwarf measurements. Since GALEX has a large field of view, this has the effect of moving the positions in the field of view for which sources with zero-point magnitudes correspond to a true 1 count $\mathrm{s}^{-1}$ at the detector. For the most part this is not a major consequence 
since the flat-field peak-to-valley variations for most of the useful field amount to about a factor of 2 even in FUV, and just 15\% in NUV. There is thus only a modest effect on the estimation of the true expected noise for a given source with field position. In order to estimate any systematics resulting from application of the new flat field, a large sample of sources common to both the GR1 and GR2/GR3 releases have been compared. We find that on average sources in GR2/GR3 are shifted 0.04 and $0.01 m_{\mathrm{AB}}$ fainter in FUV and NUV, respectively, compared to GR1. One must of course be careful applying these figures to any individual measurement because the flat has changed shape as well as its average value.

We have measured a drift in GR2/GR3 photometry of $0.003 \pm$ $0.001 \mathrm{mag} \mathrm{yr}^{-1}$ FUV and $0.016 \pm 0.0005 \mathrm{mag} \mathrm{yr}^{-1} \mathrm{NUV}$. This was identified with two independent means. In the first method (yielding the quoted results), we compared the GR2/GR3 photometry of 197 FUV and 547 NUV deep field sources between magnitudes 15 and 20 measured at approximately yearly intervals over the course of the mission. We have checked this result against the LDS749b white dwarf data; however, this second result required considerable reprocessing. By design, the GR2/GR3 sample of white dwarf measurements through 2005 shows no drift. Each measurement was given equal footing in order to improve the spatial sampling, which only included 20 measurements per year early in the mission. In 2005, we identified the NUV white dwarf saturation effect in low gain areas and also recovered the FUV detector from a major anomaly. In light of these circumstances, we implemented an extensive recalibration campaign. Over 1500 NUV measurements were added, but only 137 in the FUV because the detector was still completing its recovery. Thus, the GR2/GR3 flat, generated with all of the white dwarf samples from the mission through 2005, is biased to 2005 in both bands and very strongly so in NUV. However, areas of the flat field sampled only in, say, 2003, will reflect the proper calibration for that epoch, which means that there is an additional systematic built into the flats for isolated areas. To address the drift, we reprocessed all of the GALEXLDS749b white dwarf data using a new flat field based on a grid of 165 LDS749b field measurements made over 2 days in 2006 . We used a nearby field star of moderate NUV count rate rather than the bright LDS749b to generate the NUV flat (for FUV we used the white dwarf), mitigating concerns about the NUV saturation. The results of the white dwarf and deep field analyses are consistent with each other and are presented together in Figure 3.

The data are consistent with a linear drift (although less certain in the FUV band). We define the time of zero correction to be at eclipse 12,000 based on the weight of that epoch in generating the GR2/GR3 flats.

We have not included an FUV LDS749b data point for 2005 in Figure 3. This is because of a second issue we discovered during this analysis, which is that the 2005 FUV white dwarf data read bright by about $5 \%$ when reprocessed with the 2006 single-epoch flat field. This is a result of the way the measurements were made in 2005 while the FUV detector was completing its recovery from an anomaly. During that period (approximately a month), the detector was being cycled on and off for only very short observation periods ( $\sim$ minutes) while its stability was being tested. Since the detector was ramped to high voltage literally minutes before the observation began, it was still warming up during the observations, offsetting the calibration slightly. This means that there is a field-dependent systematic built into the GR2/GR3 FUV flat field of approximately $5 \%$ in the sense that most (about 77\%) objects are actually brighter in the FUV than they appear. NUV is unaffected.

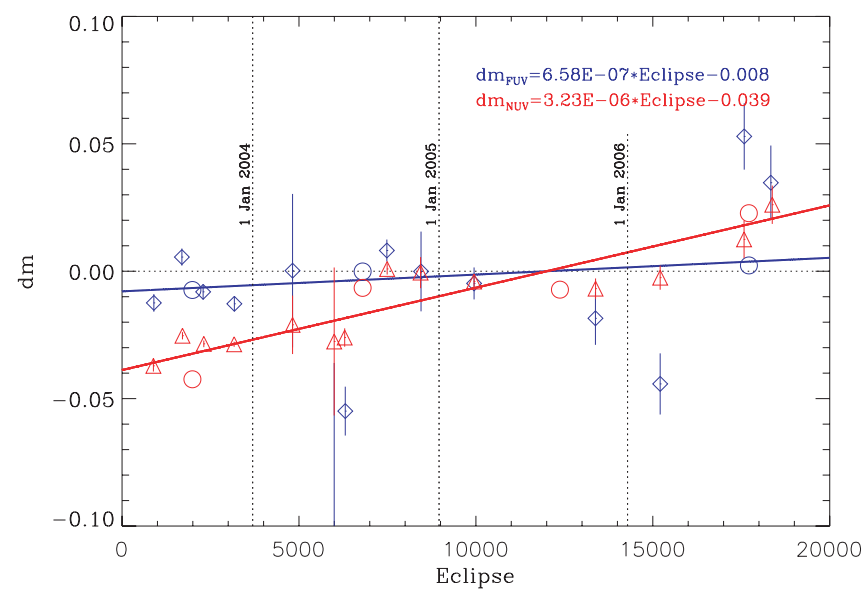

FIG. 3.- Measured photometric drift for deep field sources between magnitudes 15 and 20 over the course of the GALEX mission. FUV data are shown in blue: diamonds for the deep field sources and circles for LDS749b. NUV data are shown in red: triangles for the deep field sources and circles for LDS749b. The deep field data shown represent the mean binned in 1000 eclipse segments.

The drift is defined to be zero at eclipse 12,000 , which occurred in summer 2005 during the extensive white dwarf observing campaign on which the current calibration is mostly based. It is not clear if the trend is due to contamination in the instrument, or due to a mild global gain decrease (fatigue) in the detector microchannel plates.

We have not determined if the observed drift is due to contamination or a global MCP fatigue. Since the performance of each MCP pore is sensitive to its total exposure, We would expect fatigue to affect NUV more since it typically detects photons at about 10 times the FUV rate. Furthermore, contamination would normally be expected to preferentially affect the FUV band. On the other hand, the effect we observe appears to affect bright and dim objects the same. Whichever effect is at play, we expect to apply a correction automatically in the pipeline during a future calibration update; however, it is not accounted for in GR2/GR3.

\subsection{Relative Precision}

For accurate and repeatable measurements of unresolved sources, aperture magnitudes are usually best since they are fixed from measurement to measurement. One needs to take care to choose an appropriate aperture for the task at hand. Smaller apertures are more useful for unresolved sources, but larger ones become desirable, even for point sources, when comparing the photometry of objects measured at the center and edge of the field (where the PSF is degraded), or for bright objects that have saturated cores. For the purposes of this paper, the SExtractor MAG_APER_4 magnitude is used unless otherwise noted. This $12^{\prime \prime}$ diameter aperture is a good compromise between the very large apertures required to gather almost all of the GALEX flux in the extended PSF (as is done for the white dwarf calibration) and the small ones that are optimal for minimizing the contribution of background. The relationship between the various standard SExtractor aperture magnitudes and the large tailor-made apertures used for white dwarf calibration are presented in Figure 4.

In the GR2/GR3 release, the scatter in white dwarf measurements has been reduced to about $0.02 \mathrm{mag}$ rms in NUV and $0.045 \mathrm{mag}$ rms in FUV. This compares to a scatter of order $0.1 \mathrm{mag}$ in both bands for the GR1 release. We attribute the improvement in precision to the way the flat field has been generated, using white dwarf measurements to modify the flat locally rather than using the average to scale a sky background field. As with any instrument, care should be taken when interpreting the magnitudes of objects expected to have significantly biased spectral content 


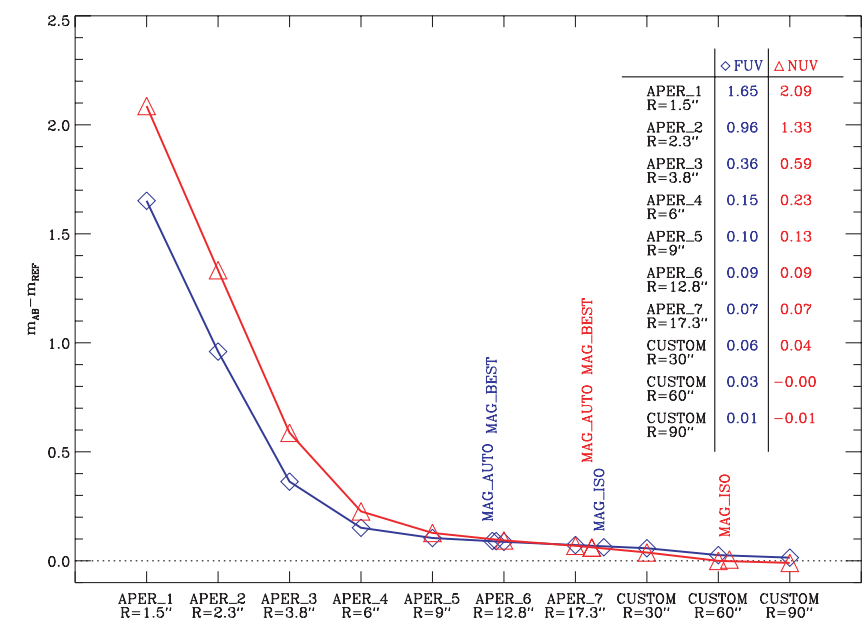

FIG. 4.-GALEX photometric "curve of growth," which shows the median magnitude offsets relative to the largest aperture used for the white dwarf LDS749b. NUV measurements have been restricted to high pulse height areas of the detector to minimize any effect due to saturation.

compared to the white dwarf. These may have more scatter due to optical effects across the field of view.

We have also verified the performance of the flat field by means independent of the partially saturated (in the NUV) white dwarf standard. For this purpose, we have examined the sample of data from the DIS described in $\S$ 4.2. This comprises a series of fields with several dozen full-eclipse-length observations, many at different roll angles (thus sampling multiple detector locations with the same source). By matching compact ( $\leq 10^{\prime \prime}$ FWHM) sources with no neighbors closer than $12^{\prime \prime}$ in the co-added catalogs to the individual visit catalogs, a visit database of sources and magnitude errors (with the co-added catalog considered to be the truth catalog) has been generated. A comparison of high- and low-gain detector measurements for a given source reveals the count rate required to cause local saturation. For both detectors, the onset of saturation is at approximately $m_{\mathrm{AB}} \sim 15$ (corresponding to 34 counts $\mathrm{s}^{-1}$ FUV and 108 counts $\mathrm{s}^{-1} \mathrm{NUV}$ ) in the lowest-gain regions of the detector. We thus restrict further analysis in this section to the range $15 \leq m_{\mathrm{AB}} \leq 17$.

With the unsaturated magnitude range determined over the whole field, we can proceed to compute the photometric repeatability, as shown in Figure 5. Here we present histograms of the $12^{\prime \prime}$ diameter aperture magnitude errors in each band. The Poisson error associated with each detection is of order $0.5 \%-1.5 \%$, while the total scatter we measure in the bright deep field sources is $\pm 0.05 m_{\mathrm{AB}}$ rms in FUV and $\pm 0.03 m_{\mathrm{AB}} \mathrm{rms}$ in NUV. These figures agree well with the white dwarf results, thus concerns about the (mild) LDS749b NUV saturation correction are reduced. If the white dwarf saturation had not been properly accounted for, the flat field would have been adversely affected in the NUV lowgain areas, which would in turn increase the photometric scatter of dimmer (definitely unsaturated) deep field sources in those same regions, and thus we would not expect good agreement in the scatter between the white dwarf and deep field samples.

The deep field visit magnitude errors are also presented as a function of the co-added "truth" magnitude in Figure 6, which serves to illustrate an interesting bias effect on the faint end of the $1500 \mathrm{~s}$ depth MIS. Here the predicted $3 \sigma$ errors (including the flat-field-limited values derived for bright sources) are overplotted, showing good agreement with most of the observed distribution but failing on the faint end. This effect is similar in magnitude but

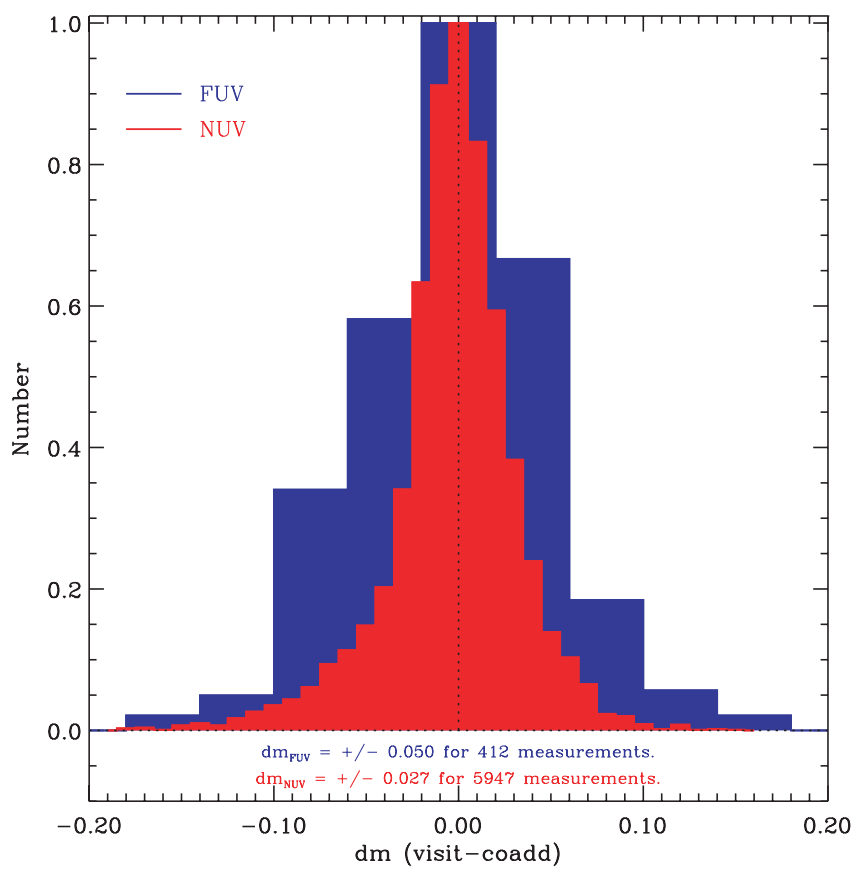

FIG. 5.- GALEX photometric precision as determined by repeated measurements of bright but unsaturated sources in the GALEX deep fields. These measurements are in good agreement with the results determined directly from repeat measurements of the white dwarf calibrators.

opposite in direction for each detector. In the FUV, sources are detected in the co-added image that are not detected in the visit images except when the Poisson errors tilt the detection to the bright side (the Eddington bias); thus, faint FUV detections are biased bright by about $0.1 m_{\mathrm{AB}}$ compared to the "truth" catalog. Similarly in the NUV, more sources are detected in the co-added image; however, the effect of the undetected sources is to force an overestimate of the NUV background of about $5 \%$ (with large scatter). In this case, faint NUV detections are biased dimmer by about $0.1 m_{\mathrm{AB}}$ compared to the "truth" catalog. In each panel, solid overplotted lines show the median of the magnitude errors in each magnitude bin, while dot-dashed lines show the same quantity after the visit magnitudes have been recomputed using the co-add background estimation. This correction is indistinguishable from the solid line median in the FUV panel because the background is negligible, but completely cancels the faint end bias in the NUV. Since the effects of these two biases are in opposite directions for each band, they have a more significant effect on the UV color, about $0.2 m_{\mathrm{AB}}$ at the faint end of the distribution.

For completeness, we present a similar analysis of the photometric errors in the $100 \mathrm{~s}$ AIS sample relative to cross-matched sources in overlapping MIS fields. As illustrated in Figure 7, we find that the AIS magnitudes are biased bright at the faint end relative to the MIS for both detectors and that corrections to the AIS magnitudes using MIS backgrounds have no effect at all. This result stems from the shallower depth of the AIS survey, which is source-limited rather than background-limited.

\subsection{Linearity}

There are two sources of photometric nonlinearity in the instrument: global nonlinearity resulting from the finite period required for the electronics to assemble photon lists (during which time new events are locked out) and local nonlinearity resulting 

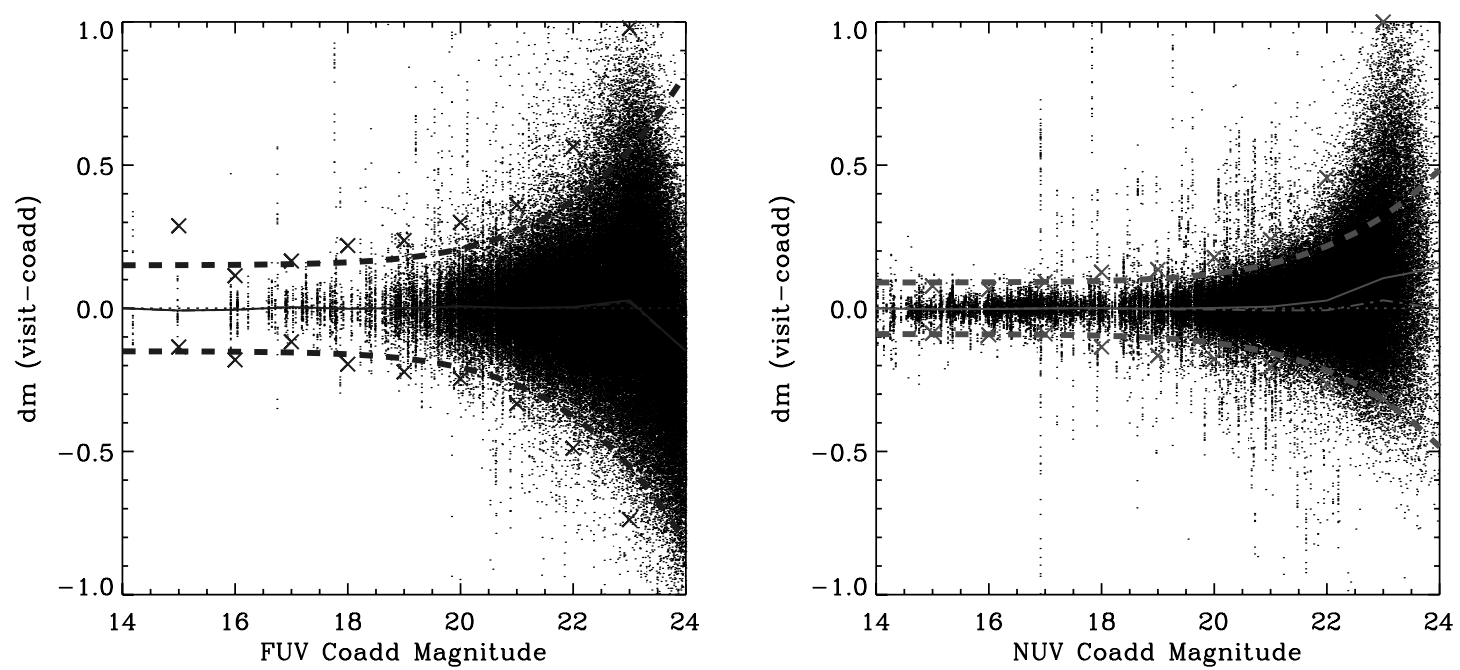

FIG. 6.-GALEX photometric repeatability as a function of magnitude as determined by repeated measurements of sources in the GALEX deep fields (with individual measurements of order $1000 \mathrm{~s}$ ). In each panel, heavy dashed lines are the $3 \sigma$ values that would be expected given the flat field precision and Poisson counting statistics, while the crosses show the actual value for each magnitude bin. Solid overplotted lines show the median of the magnitude errors in each magnitude bin, while dot-dashed lines show the same quantity after the visit magnitudes have been recomputed using the co-add background estimation. [See the electronic edition of the Supplement for a color version of this figure.]

from the MCP-limited current supply to small regions around a bright sources. The local effect complicates the NUV white dwarf calibration measurements.

Global effects are easily measured using the on-board "STIM" pulser, which electronically stimulates each detector anode with a steady, low rate stream of electronic pulses. These are resolved as point sources in the corners of the dose (-scdose ) images. Since the real rate of STIM pulses is accurately known, the measured rate is used by the pipeline to scale the effective exposure. The count rates for all sources in the field are simultaneously corrected. This effect is typically about $10 \%$ in NUV and a few percent in FUV; however, it can become quite significant $(\sim 50 \%)$ for the brightest fields.

Local nonlinearity cannot be corrected well with the existing calibration. It affects not only the measured count rate of an in- dividual bright source but also the source shape and position. Furthermore, the effects vary around the field of view with the gain of the detector (as exemplified by the white dwarf saturation correction described in $\S 4.1$ ). We have used standard stars to estimate the local nonlinearity in each band, as shown in Figure 8 . These data may be used to approximately correct bright sources that are within the measured range. Note that the measurements presented in the figure are for 34.5" (APER_7) and $3^{\prime}$ diameter apertures; the effect will be much worse when small apertures are used. The NUV detector is more robust to bright sources because its photoelectrons are proximity-focused and thus present a larger source image (with lower count density) to the MCP. Local photometric nonlinearity is strongly source-size dependent. The saturation shown in Figure 8 is a worst-case scenario (using stars). Wide scatter in measurements of bright sources is an indication
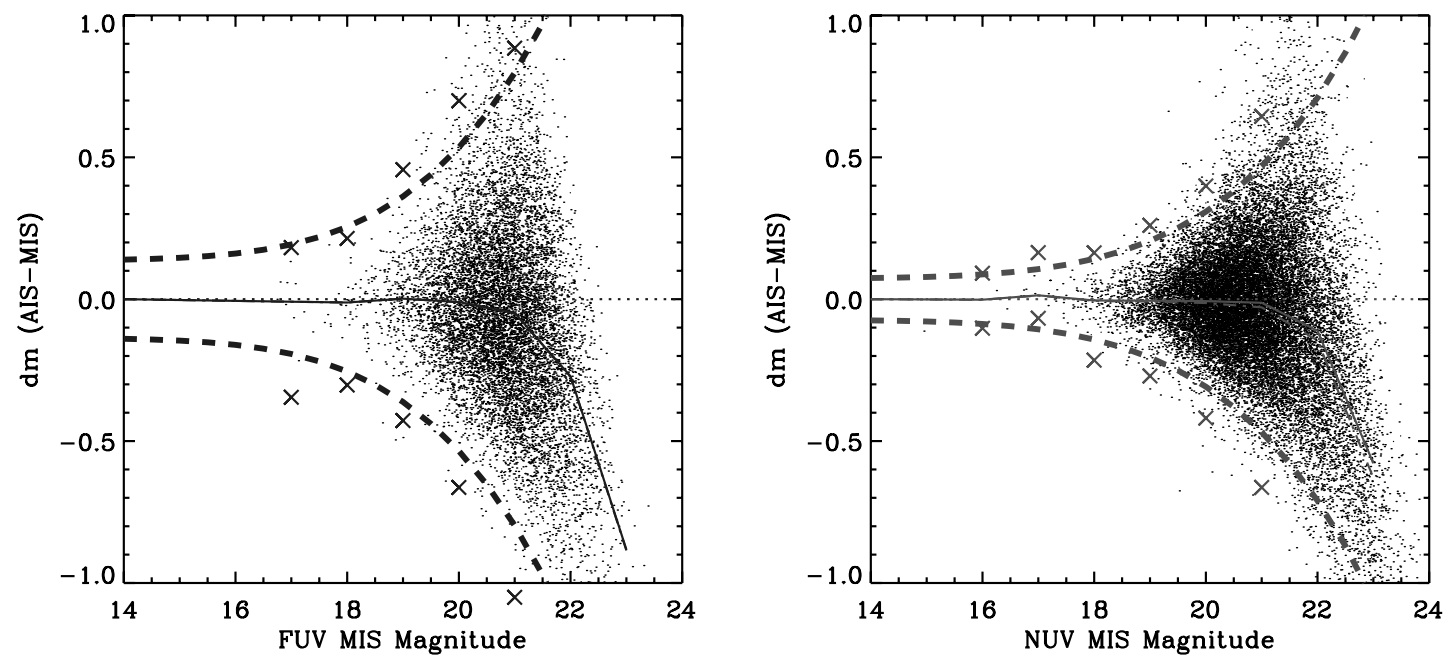

FIG. 7.-GALEXAIS photometric repeatability as a function of magnitude as determined by repeated measurements of sources in overlapping MIS fields. In each panel, heavy dashed lines are the $3 \sigma$ values that would be expected given the flat field precision and Poisson counting statistics, while the crosses show the actual value for each magnitude bin. Solid overplotted lines show the median of the magnitude errors in each magnitude bin, while dot-dashed lines show the same quantity after the visit magnitudes have been recomputed using the co-add background estimation (in the AIS this correction does not have a significant effect). At the very faint end, there is an apparent bias toward visit magnitudes brighter than co-added magnitudes due to Eddington bias in the source-limited sample. [See the electronic edition of the Supplement for a color version of this figure.] 


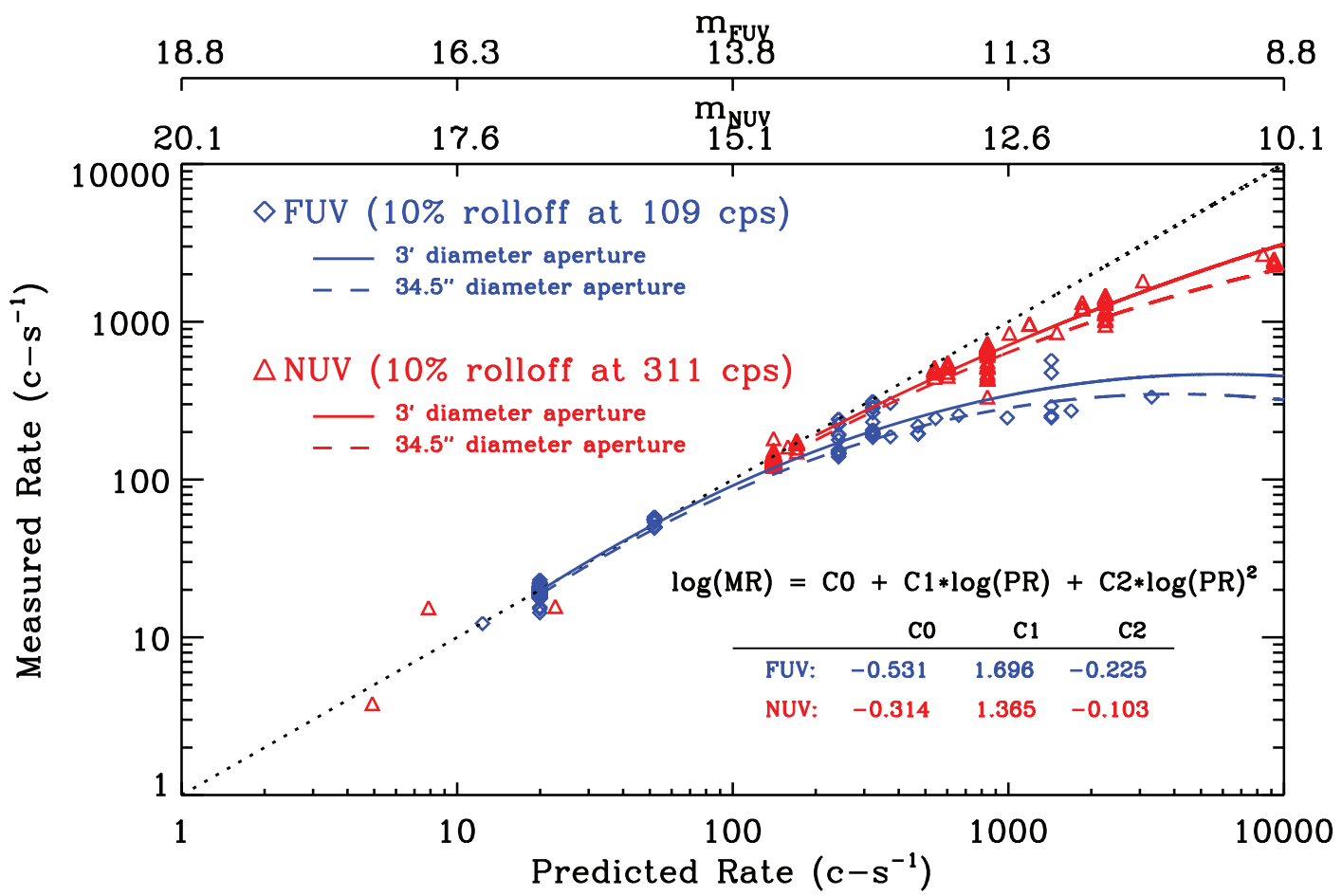

FIG. 8.- Measured count rate versus predicted count rate for the white dwarf standards measured to date by GALEX using 34.5" (APER_7) and $3^{\prime}$ diameter apertures. These measurements represent a worst case because they are for point sources. The nonlinearity will be much worse when measuring fluxes using small apertures, since this effect is concentrated at the core of each image.

of the variation of the onset of saturation at different detector locations.

We have increased the local count rate limit to 30,000 counts $\mathrm{s}^{-1}$ per source in NUV, which corresponds to approximately 9 th magnitude. This new limit allows most of the sky to be observed. The FUV local limit is maintained at 5000 counts s$^{-1}$, which is not currently a survey constraint. Global detector limits are set in hardware (rather than the planning system) to 100,000 counts $\mathrm{s}^{-1}$, a limitation based largely on the boundaries of ground testing and engineering judgment about safe rates of gas generation inside the sealed detector tubes. Areas such as the Galactic plane and the Magellanic Clouds are still restricted by the 100,000 counts $\mathrm{s}^{-1}$ global limit.

\subsection{The GALEX Deep Fields}

The specific case of faint DIS photometry presents its own special challenges. The relatively large GALEX PSF affects the photometry of faint sources, mostly due to source confusion (Xu et al. 2005). As an alternative to the GALEX pipeline for faint source extraction, a Bayesian approach has been attempted by others (Guillaume et al. 2006) to estimate the UV flux using prior information from external, well-resolved images (mostly from visible wavelengths). The basic idea is to generate an image with prior sources convolved with the GALEX PSF and to estimate what scaling factors to apply that will best fit the UV image. The implemented method is based on a maximum likelihood parametric optimization in the presence of Poisson noise. Scaling factors were extracted via an iterative procedure to solve the set of nonlinear equations based on the expectation-maximization (EM) method. In order to test the reliability of the photometry extracted using this method, simulated objects (with a large range of UV flux) were added to the GALEX DIS images. For an NUV DIS image with $60 \mathrm{ks}$ exposure, it was possible to recover the photometry of simulated sources with an accuracy of $0.3 m_{\mathrm{AB}}$ to NUV
25.5, extending the faint-end limit of the survey by about a magnitude.

\section{RESOLUTION}

There are numerous contributors to the width of the GALEX PSF, including the optics, detectors, and ground pipeline. The reconstruction algorithms for GR2/GR3 have improved to the point that the instrumental contributions are now the largest share, particularly in the proximity-focused NUV channel. As we will show, the GALEX detectors dominate in this area and have significantly position-dependent performance.

To verify the fundamental instrument performance from onorbit data, some bright stars were analyzed individually, outside the pipeline. These results show performance that is consistent with or better than what was measured during ground tests. We have also verified the end-to-end performance including the pipeline by stacking images of stars from the MIS survey that were observed at different locations on the detector. The results of these composites are shown in Figures 9 and 10. Performance is reasonably uniform except at the edge of the field, where it is significantly degraded. Some remaining degraded areas of the images may yet be improved with updates to the static calibration. Note that DIS surveys co-add data with many roll angles, so the DIS PSF is considerably more symmetrical across the field of view.

Many of the regions with degraded resolution correlate with low detector gain, an effect illustrated in Figure 11. The relative detector gain (or charge, "Q") in the region of measurement for each source is tabulated in the merged catalog. This gain measurement is obtained from a lookup table that represents the detector in an unsaturated condition. The actual gain measured during the observation is available in the pulse height (-scq) image data product.

It is also evident that the GALEX PSF has structure a significant distance from the center. We have characterized this by building a 


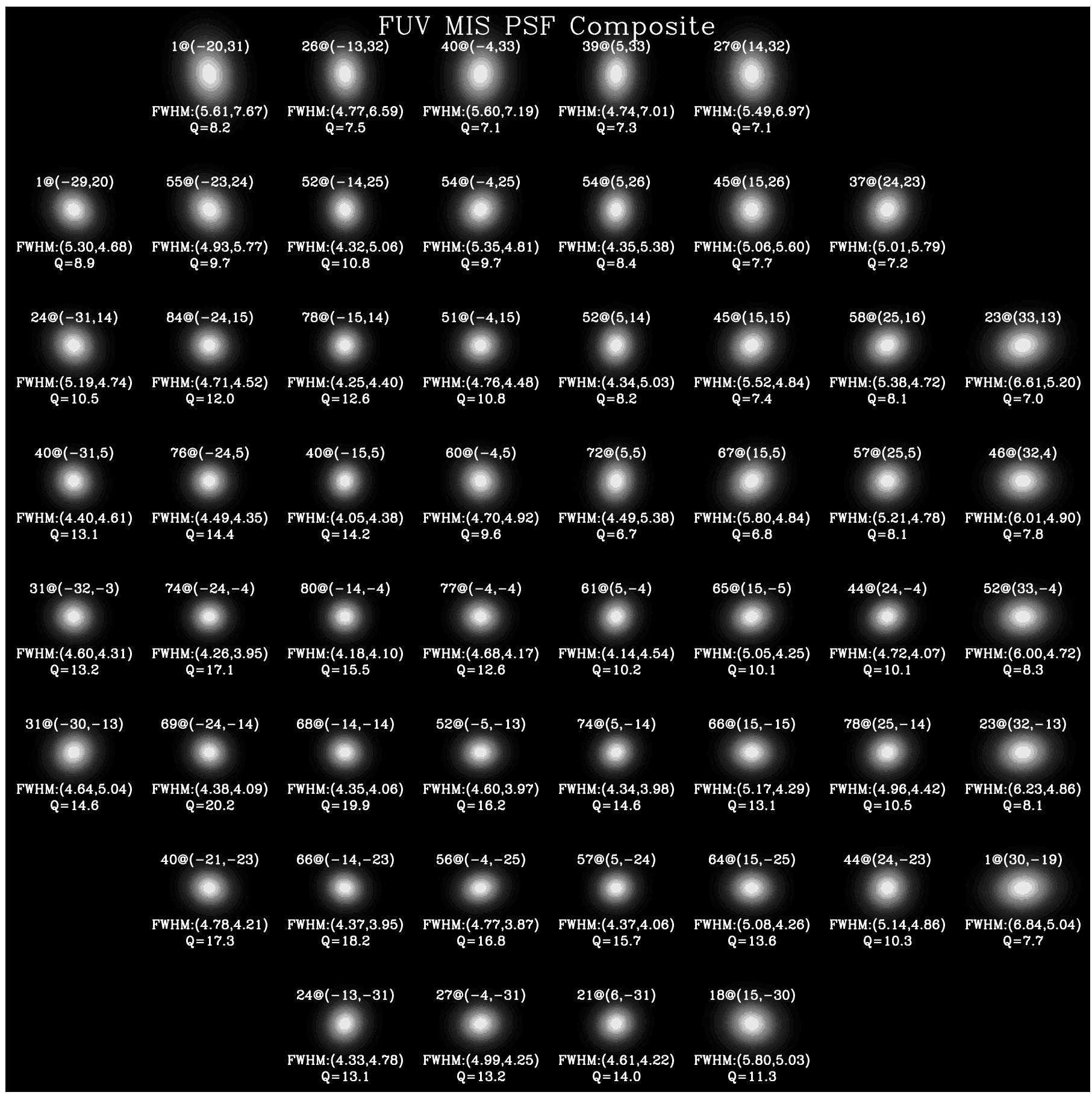

FIG. 9.-Composite PSFs for the GALEX FUV channel in detector coordinates. The individual images are exaggerated for clarity. Upper data values in the format $N @(X, Y)$, where $N$ is the number of stacked images and $(X, Y)$ is the position in minutes of arc from the field center. The FWHM is specified in arcsec for each detector axis. $Q$ is the typical pulse height at the specified detector location, in bins. The pulse height scale is not extremely well calibrated, but it is linear with 10 bins $\sim 1 \times 10^{7}$ electrons). [See the electronic edition of the Supplement for a color version of this figure.]

composite PSF of the white dwarf calibrator LDS749b, as shown in Figure 12. The effects of local nonlinearity are also illustrated here by the highly saturated white dwarf $\mathrm{HZ} 44$, the brightest we have observed with GALEX in imaging mode. The effect of the saturation is strongest in the core of the PSF, causing the wings to be strongly emphasized.

\section{ASTROMETRY}

The GALEX GR2/GR3 astrometric precision represents an improvement of order $20 \%$ over GR1 (and much more compared to the Early Data Release). The dynamic pipeline transformation is generally not a limiting factor as it had been in earlier releases. The static transformation (primarily the distortion map) is also performing very well for most of the field of view. Improvements could still be made that would not only improve the global astrometric precision but also the point spread function (PSF) at the edge of the field.

The distortion maps for the GR2/GR3 calibration are the result of several flight-based iterations on the preflight calibration. The first, which was implemented for the GR1 data release, used 


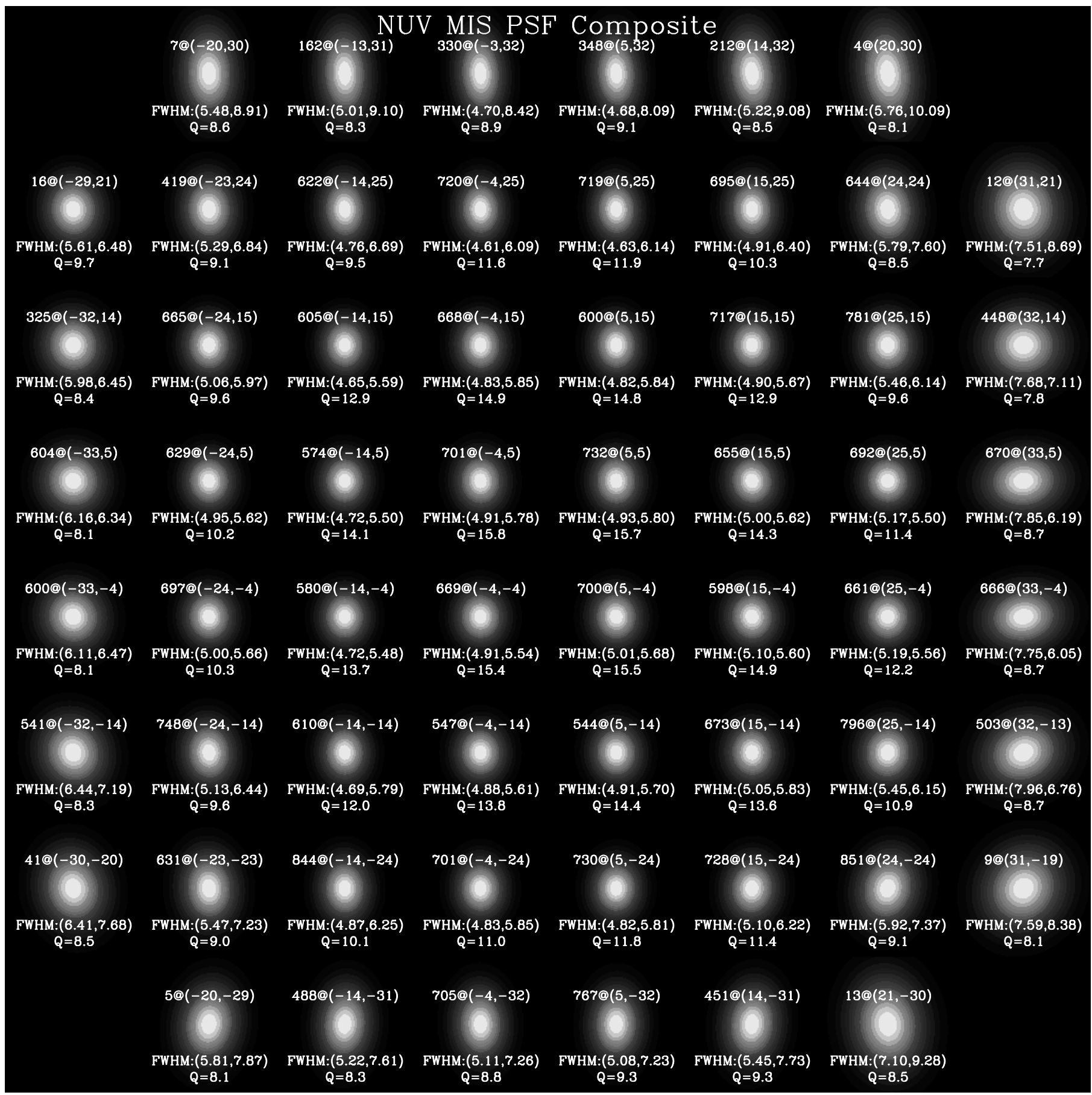

FIG. 10.-Composite PSFs for the GALEX NUV channel in detector coordinates. The individual images are exaggerated for clarity. Upper data values are $N @(X, Y)$, where $N$ is the number of stacked images and $(X, Y)$ is the position in minutes of arc from the field center. The FWHM is specified in arcsec for each detector axis. $Q$ is the typical pulse height at the specified detector location, in bins. The pulse height scale is not extremely well calibrated, but it is linear with 10 bins $\sim 1 \times 10^{7}$ electrons). [See the electronic edition of the Supplement for a color version of this figure.]

Tycho-2 catalog (Hog et al. 2000) reference stars to refine the distortion map for each detector independently. An iterative technique was used until the measured GALEX positions converged with the reference positions, a technique that relied on the assumption that the distortion across a $\sim 1^{\prime}(\sim 1 \mathrm{~mm})$ region of the detector (the footprint of the dither pattern) is reasonably represented by a single average value. This technique worked well; however, there were relatively few FUV stars available to perform the analysis (a few thousand). The FUV-NUV relative as- trometry retained some noticeable $\left(\sim 1^{\prime \prime}\right)$ misalignments. We have addressed this issue with cross-matched SDSS data. Since the SDSS astrometry is better than $0.1^{\prime \prime} \mathrm{rms}$ (Pier et al. 2003), it can be regarded as essentially perfect for our purposes. The sample we gathered contains 69642 objects spectroscopically classified as quasars (SDSS specClass $=3$, a convenient point source sample compiled for other applications). Objects were considered matches if they fell within $2.5^{\prime \prime}$ of each other. There was no discrimination based on location in the GALEX field of view. We 


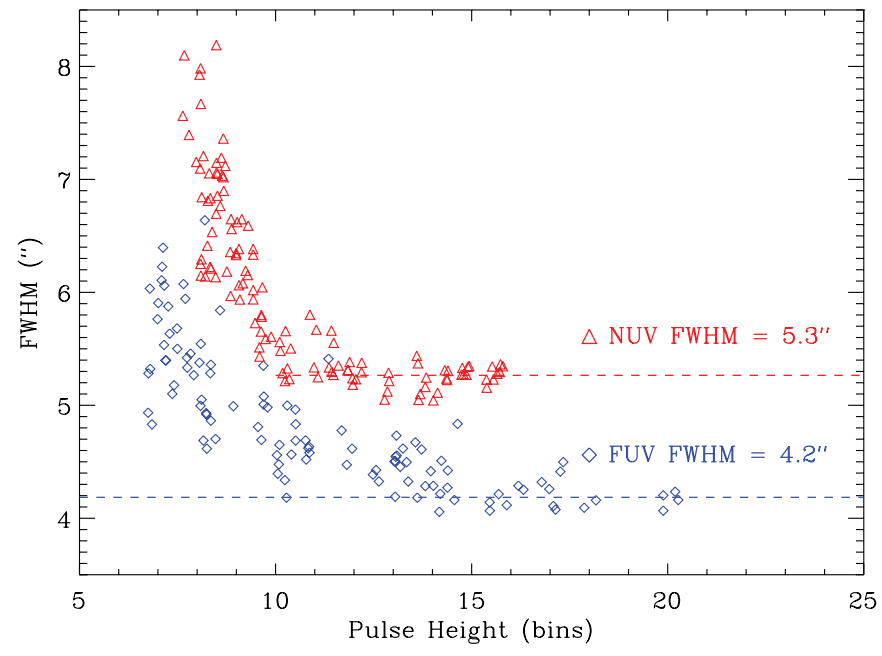

FIG. 11.-Full width at half-maximum of the GALEX PSF based on composite images for each detector as a function of intrinsic detector pulse height. In general, low pulse height regions are near the edge of the detector.

binned these results into a relatively coarse grid $\left(\sim 1^{\prime}\right)$, the intent of which was to "stretch" the existing plate solution to remove low spatial frequency errors. The result of this improvement is that the GR2/GR3 absolute astrometric precision achieved by the GALEX MIS within $0.6^{\circ}$ of the field center for bright (signalto-noise ratio $\geq 10$ ) point sources is $0.49^{\prime \prime} \mathrm{rms}$ in the NUV and $0.59^{\prime \prime} \mathrm{rms}$ in the FUV, with similar relative precision between the two bands.

In Figure 13 we present histograms of the position errors for high S/N SDSS quasars detected by GALEX. The most noticeable image defect resulting from the current astrometric solution is the degraded PSF at the edge of the field. As shown in Figures 14 and 15, the astrometric precision in the outer $5^{\prime}$ of the detector, while degraded, still performs nearly at the level of the central $30^{\prime}$. Generally speaking, matches between GALEX and other surveys can be safely performed with a $2^{\prime \prime}$ window, as indicated by Figure 16.

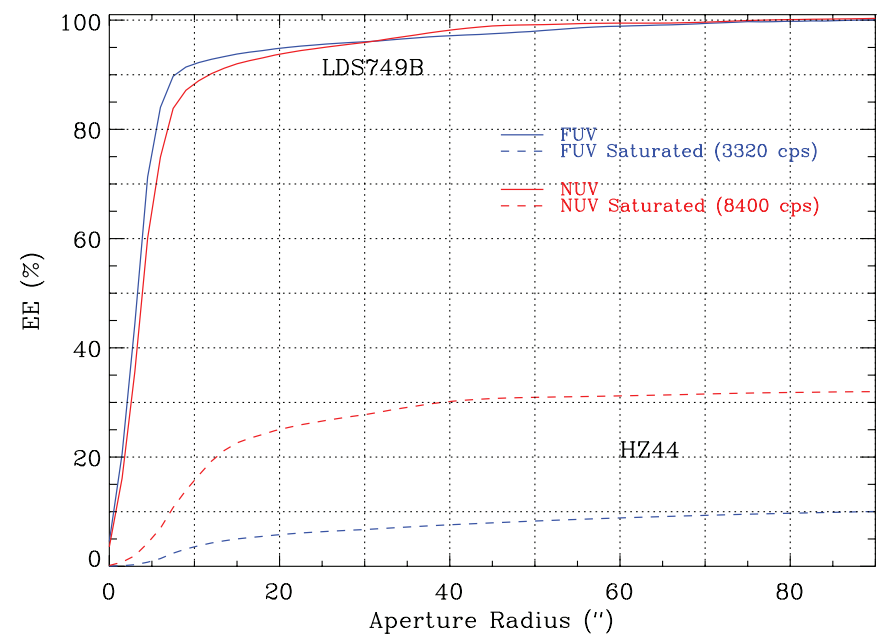

FIG. 12.-GALEX FUV and NUV composite encircled energy curves for two white dwarf standards. A composite of LDS749b measurements forms the unsaturated (solid) curve, while the brightest standard that we have observed, HZ 44, defines the saturated curve. The reference count rates for the saturated curves are the predicted values; measured rates are much lower, as indicated by the low encircled energy values.

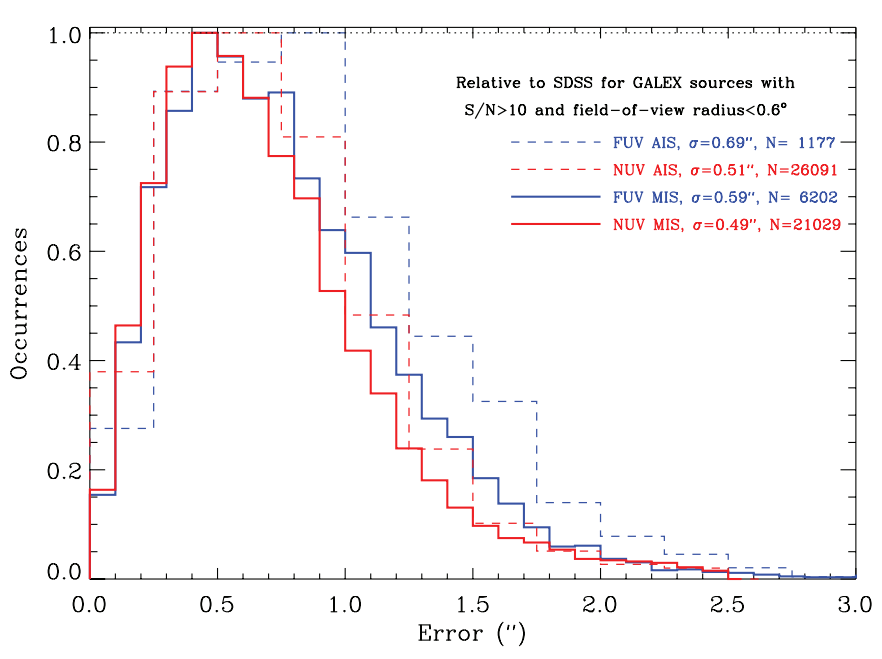

FIG. 13.-Distribution of absolute astrometric errors in the GALEX GR2/GR3 survey data for quasars with $\mathrm{S} / \mathrm{N} \geq 10$ in each band matched against the SDSS.

The most promising future improvement to the astrometric calibration probably involves a new pipeline module that would scale the amplitude of the distortion maps by a factor determined from measurements of the STIM pulses at the corners of each image. Much of the necessary data to eliminate this "breathing" mode is already available. Another more complex possibility involves reprocessing the reference star data in $\sim 20 \mathrm{~s}$ intervals to reduce the footprint of each source sample on the detector and thus more finely sample the detector grid. Such an analysis would be most useful near the edge of the detector where the distortion map is changing rapidly.

\section{SPECTROSCOPY}

The spectroscopic mode utilizes a $\mathrm{CaF}_{2}$ grism (a prism with a ruled surface) that can be moved into the converging beam of the telescope to form simultaneous (slitless) spectra of all sources in the field in both bands. Many spectra will normally overlap, a problem addressed by rotating the grism on its axis to different position angles during repeat observations of the same field. Each spectrum has an "undeviated wavelength point" around which it will rotate as the grism position angle is changed (thus, any given

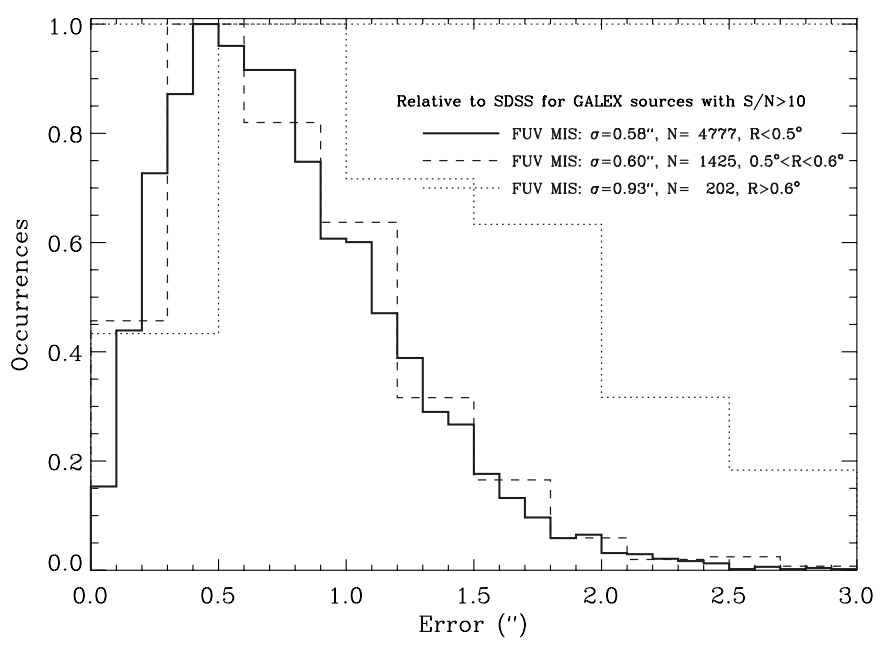

FIG. 14.-FUV GR2/GR3 MIS absolute astrometric performance for field center and field edge distributions of SDSS quasars with GALEX $\mathrm{S} / \mathrm{N} \geq 10$. [See the electronic edition of the Supplement for a color version of this figure.] 


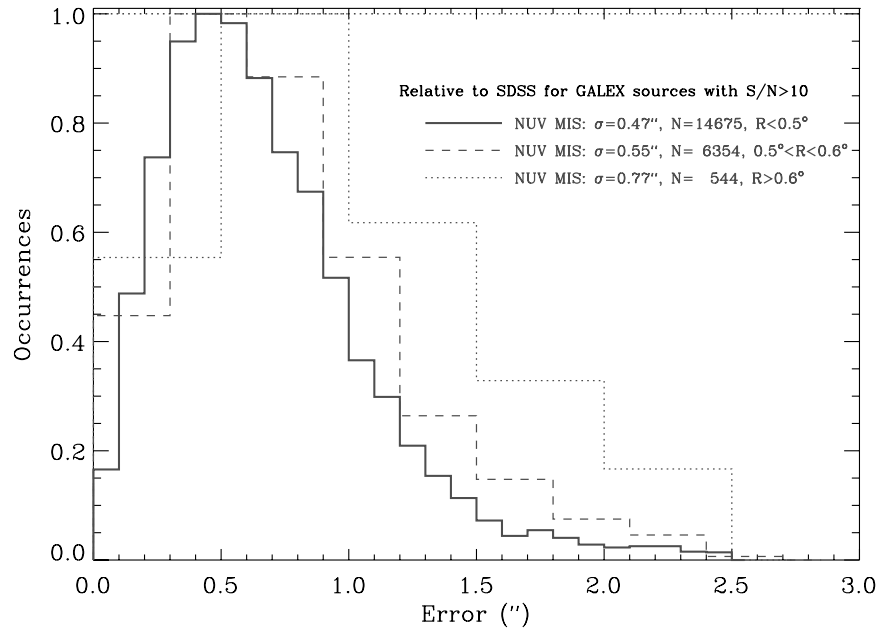

FIG. 15.-NUV GR2/GR3 MIS absolute astrometric performance for field center and field edge distributions of SDSS quasars with GALEX S/N $\geq 10$. [See the electronic edition of the Supplement for a color version of this figure.]

spectrum will have an optimal set of grism position angles depending on the location of nearby sources). As the position angle changes, the undeviated wavelength point remains fixed in the field as illustrated by Figure 17. The resulting spectra can be combined to eliminate confusion.

The standard GALEX spectroscopy pipeline is designed for isolated point sources, which are detected in the GALEX direct image observations. Extended or overlapping sources, or sources that do not appear in the direct image catalog, require a special pipeline reduction with modified parameters.

A relative position correction as a function of time is applied to the spectral image data using a technique very similar to that used for direct image photons. Using the brightest sources from the catalog, an aspect correction is applied to the entire exposure by fitting four parameters: shift in dispersion direction, shift in spatial direction, spacecraft roll angle, and grism position angle. A spectrum points north (blue to red) when the grism position angle is $0^{\circ}$ and toward the east when it is $90^{\circ}$.

Bright sources may show grism orders ranging from -4 to +5 across the field; however, only two orders in each band are typ-

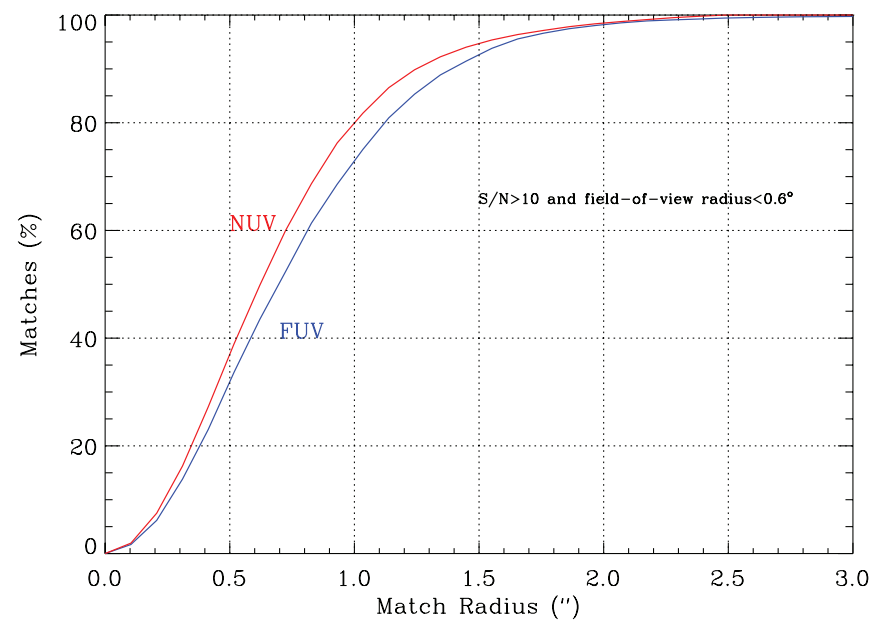

FIG. 16.-Percentage of GALEX-SDSS matches as a function of matching radius. An MIS sample with $\mathrm{S} / \mathrm{N} \geq 10$ and field radius less than $0.6^{\circ}$ was used for this analysis. Note that the matched sample is limited to $2.5^{\prime \prime}$ maximum error, although based on the shape of the distribution this does not appear to be an issue.

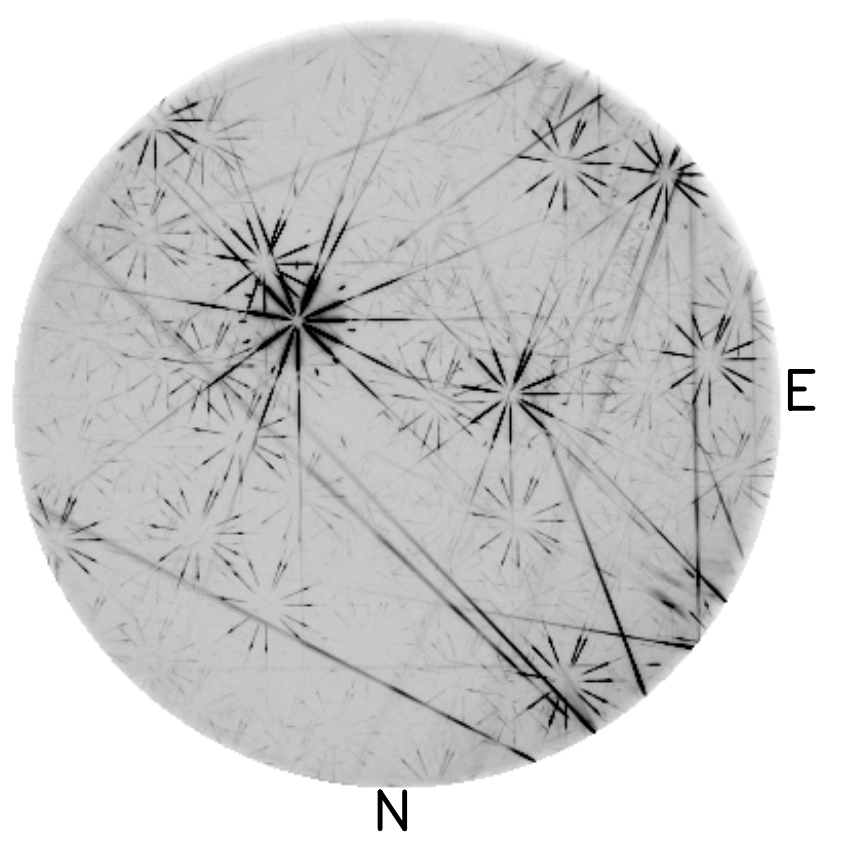

FIG. 17.-Composite image of GALEX grism spectra collected with multiple grism position angles. As the grism is rotated in its mount, each spectrum spins around its own undeviated wavelength point to form a "pinwheel" in the composite, illustrating the optimal grism angle (producing an unconfused spectrum) for each source.

ically extracted. For NUV, the highest response is in first order with a total system peak response of $40 \mathrm{~cm}^{2}$. For FUV, the strongest order is second with a peak response of $20 \mathrm{~cm}^{2}$. The dispersion angles $\gamma_{\mathrm{FUV}}$ and $\gamma_{\mathrm{NUV}}$ in arcseconds from the undeviated wavelength point for FUV second order and NUV first order are given by

$$
\begin{aligned}
\gamma_{\mathrm{FUV}}= & -4530.7+7.3859 \lambda-3.9254 \times 10^{-3} \lambda^{2} \\
& +7.4750 \times 10^{-7} \lambda^{3}, \\
\gamma_{\mathrm{NUV}}= & -882.1+7.936 \times 10^{-1} \lambda-2.038 \times 10^{-4} \lambda^{2} \\
& +2.456 \times 10^{-8} \lambda^{3},
\end{aligned}
$$

where $\lambda$ is the wavelength in $\AA$, accurate to a few tenths of an arcsecond.

Using the catalog positions, image strips (aligned to the dispersion direction) are created for each source using the corrected photon positions. Figure 18 shows how a portion of the field image translates into a dispersion-aligned image strip for each band. The figure shows image strips covering $1700^{\prime \prime}$ in the dispersion direction to illustrate the weaker orders. Only $900^{\prime \prime}$ (from $-100^{\prime \prime}$ to $+800^{\prime \prime}$ relative to the undeviated point) covering the two strongest orders in each band are stored during actual data reductions. The width of each image strip is $78^{\prime \prime}$.

Figure 18 also shows the undeviated point in each image strip (marked with a cross) that occurs at about $1700 \AA$ in first order in both bands. This is the point where the spectral images for all position angles intersect.

The spectral shape, strength, and position of neighboring sources are estimated from the direct image catalog and are masked from each image strip. Additional masking is done on each strip when the exposures are co-added to eliminate other artifacts not predicted by known neighboring sources, which may be due to reflections, ghosts, hot spots, or unidentified sources near the edge of 

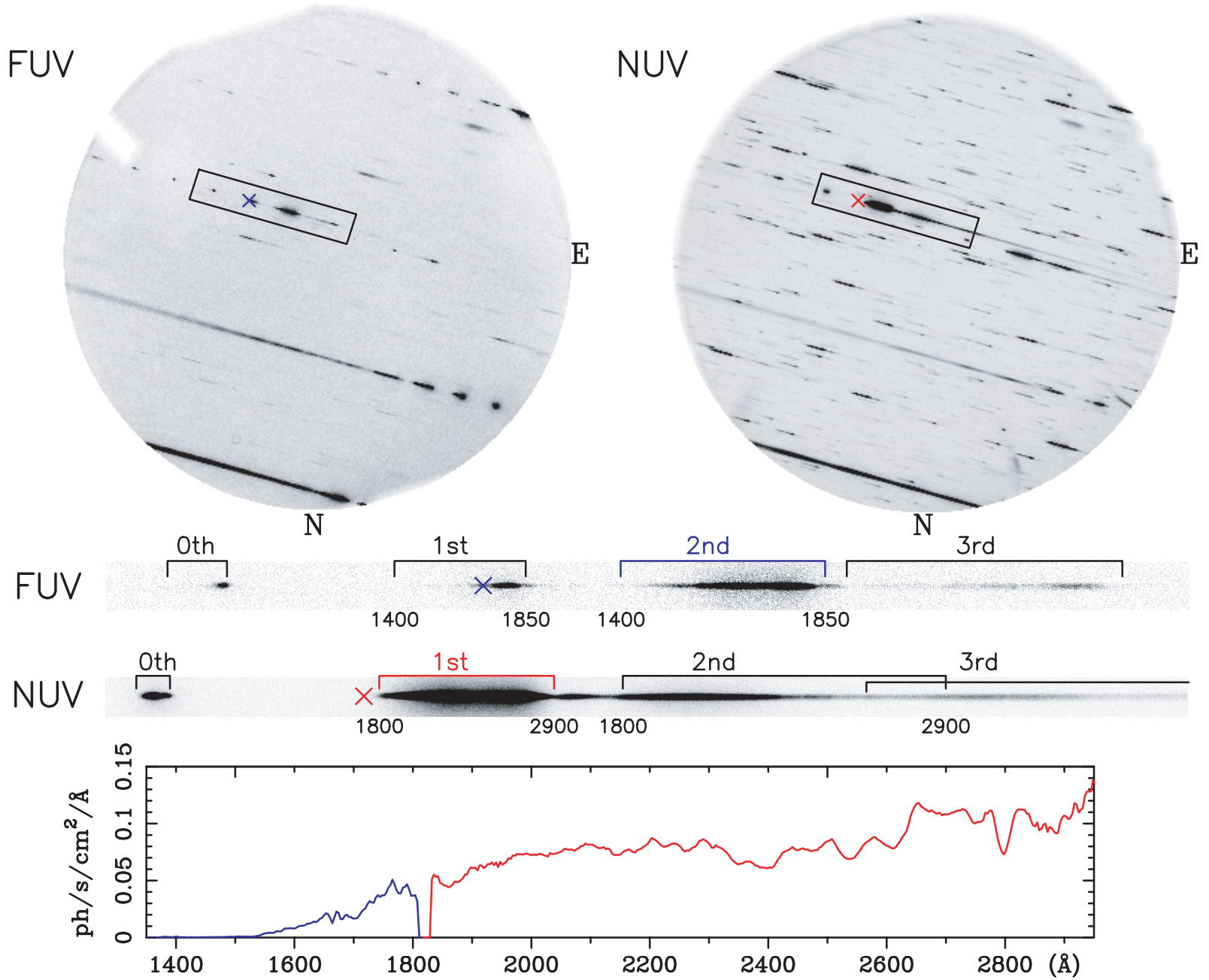

FIG. 18.-Example bright object GALEX spectra. Circular images at top show the reconstructed FUV and NUV spectral image data. A rectangular region of interest identifies the same spectrum in each band, while a cross marks the undeviated wavelength point around which the spectra would spin if the grism were rotated. The rectangular regions are enlarged and annotated in the middle of the frame, where wavelength ranges and spectral orders are identified. In the bottom panel, the calibrated spectrum is shown in blue for the FUV portion and red for the NUV portion.

the field. The response map is then applied and the background is fitted and subtracted at each wavelength point. An aperture extraction (summation) is computed for the primary spectrum using the brightest order in each band (NUV first and FUV second). An optimal extraction is also computed using a PSF model. A $20 \AA$ region is set to zero in the spectrum at around $1820 \AA$, indicating the separation between FUV and NUV.

A list of sources for which spectra will be extracted is determined by estimating what the final $\mathrm{S} / \mathrm{N}$ of a given source will be after co-adding all grism observations. Any source which will achieve a $\mathrm{S} / \mathrm{N}$ per resolution element in the co-added result of $\geq 2$ in FUV or $\geq 3$ in NUV is selected for spectral extraction. For multiexposure data reductions, the masked image-strip photon and response data are co-added for each source. For both individual and co-added spectra, a response correction is applied as a function of wavelength and the spectra are rebinned to a linear scale. The primary (NUV first order and FUV second order), secondary (NUV second order and FUV third order), and optimal (the primary spectrum with PSF weighting in the spatial direction) extractions are stored in 3 arrays in the final reduced data file. The estimated $\mathrm{S} / \mathrm{N}$ for unresolved sources in single-orbit spectra is shown in Table 6.
The usable wavelength range is approximately 1300-1820 for FUV (second order) and 1820-3000 $\AA$ for NUV (first order). The average dispersion is 1.64 and $4.04 \AA \operatorname{arcsec}^{-1}$ for FUV (second order) and NUV (first order), respectively. The average resolution $(\lambda / \Delta \lambda)$ for a point source (assuming a $5^{\prime \prime} \mathrm{PSF}$ ) is about $200(8 \AA)$ and 118 (20 $)$ ) for FUV (second order) and NUV (first order), respectively.

Although there is evidence that the response may vary with position on the detector or with the grism position angle, a single wavelength-dependent calibration is used for each band. These variations may introduce a calibration error of about $3 \%$ in the

TABLE 6

Typical Signal-to-Noise Ratio for Unresolved GaLEX Spectra AT MIS DEPTH (1500 s)

\begin{tabular}{|c|c|c|}
\hline$m_{\mathrm{AB}}$ & FUV S/N & NUV S/N \\
\hline 16 & 10.4 & 21.2 \\
\hline 18 & 3.4 & 6.1 \\
\hline 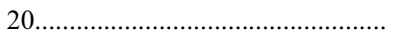 & 0.79 & 1.22 \\
\hline 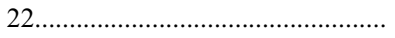 & 0.14 & 0.20 \\
\hline
\end{tabular}


body of each spectral order and about $10 \%$ near the edges of each order.

\section{CONCLUSIONS}

We have described the GALEX performance and calibration results for the GR2 and GR3 data releases. These releases have identical pipeline calibrations that are significantly improved over the previous GR1 release. The analysis we have presented provides an overview of the data quality, errors, and peculiarities that are likely to confront astronomers.

The relative photometric precision is 0.05 and $0.03 m_{\mathrm{AB}}$ in the FUV and NUV, respectively. We have identified several photometry issues that will be improved in future calibrations. The first is a systematic drift with time of order $1 \%$ in FUV and $6 \%$ in NUV, total, over the period of the released data $(\sim 4 \mathrm{yr})$. The sense of the drift is that objects appear to be growing fainter with time. The second issue is that the current FUV calibration relies on data collected in a nonstandard mode during the $2005 \mathrm{FUV}$ recovery, and these data appear bright by $5 \%$ compared to other measurements of the same objects collected in the standard mode. The effect of this is to make GR2/GR3 objects systematically fainter in the FUV than they actually are. The third issue is that the NUV zero point is defined by a white dwarf calibrator that is at least partially saturated. While we have evidence that the saturation is mild and correctable, we are planning confidencebuilding observations of an additional white dwarf, the dimmest in the CALSPEC catalog, to help resolve the issue.
The astrometric precision has been improved, by about $20 \%$, to $0.5^{\prime \prime} \mathrm{rms}$. There is evidence that it may be improved further by accounting for the detector "breathing" mode already measured with existing data from an external pulser that runs in parallel during all detector operations.

All of our performance parameters continue to meet flight requirements. As the mission continues, we naturally expect the calibration to evolve. The instrument itself continues to function nominally in spite of a series of flight anomalies especially with the FUV detector. We have modified operations and flight software over the mission to respond to these issues. While some of them have significantly affected the FUV observation efficiency, none give any indication of affecting the instrument performance. We believe there is room to increase the count rate limits for both detectors (especially FUV), and to the extent possible we plan to explore higher rate fields closer to the Galactic plane after the primary surveys are completed.

GALEX (Galaxy Evolution Explorer) is a NASA Small Explorer, launched in 2003 April. We gratefully acknowledge NASA's support for construction, operation, and science analysis for the GALEX mission, developed in cooperation with the Centre National d'Etudes Spatiales of France and the Korean Ministry of Science and Technology.

\section{Facilities: GALEX}

Bertin, E., \& Arnouts, S. 1996, A\&AS, 117, 393

Bohlin, R. C. 1996, AJ, 111, 1743

Bohlin, R. C., et al. 2001, AJ, 122, 2118

Boksenberg, A., et al. 1973, MNRAS, 163, 291

Budavari, T., et al. 2007, in ASP Conf. Ser. 376, Astronomical Data Analysis

Software and Systems XVI, ed. R. A. Shaw, F. Hill, \& D. J. Bell (San Francisco: ASP), in press

Forster, K., et al. 2006, Proc. SPIE, 6270, 627004

Guillaume, M., et al. 2006, Proc. SPIE, 6064, 606332

Hog, E., et al. 2000, A\&A, 355, L27

Jelinsky, P., et al. 2003, Proc. SPIE, 4854, 233

\section{REFERENCES}

Landolt, A. 1992, AJ, 104, 340

Martin, D. C., et al. 2005, ApJ, 619, L1

Morrissey, P. 2006, Proc. SPIE, 6266, 62660Y (see update at http://www.galex .caltech.edu/DATA/gr2_docs/Lessons_learned_SPIE_update_Morrissey.pdf)

Morrissey, P., et al. 2005, ApJ, 619, L7

Oke, J. B., \& Gunn, J. E. 1983, ApJ, 266, 713

Pier, J. R., et al. 2003, AJ, 125, 1559

Press, W., Teukolsky, S. A., Vetterling, W. T., \& Flannery, B. P. 1992, Numerical Recipes in C (2nd ed.; Cambridge: Cambridge Univ. Press)

Urban, S. E., et al. 1998, AJ, 115, 2161

$\mathrm{Xu}, \mathrm{C}$. K., et al. 2005, ApJ, 619, L11 